\title{
Systematic review of sedentary behaviour and health indicators in school-aged children and youth
}

\author{
Mark S Tremblay ${ }^{1 *}$, Allana G LeBlanc ${ }^{1}$, Michelle E Kho², Travis J Saunders' ${ }^{1}$, Richard Larouche ${ }^{1}$, Rachel C Colley ${ }^{1}$, \\ Gary Goldfield ${ }^{1}$ and Sarah Connor Gorber ${ }^{3}$
}

\begin{abstract}
Accumulating evidence suggests that, independent of physical activity levels, sedentary behaviours are associated with increased risk of cardio-metabolic disease, all-cause mortality, and a variety of physiological and psychological problems. Therefore, the purpose of this systematic review is to determine the relationship between sedentary behaviour and health indicators in school-aged children and youth aged 5-17 years. Online databases (MEDLINE, EMBASE and PsycINFO), personal libraries and government documents were searched for relevant studies examining time spent engaging in sedentary behaviours and six specific health indicators (body composition, fitness, metabolic syndrome and cardiovascular disease, self-esteem, pro-social behaviour and academic achievement). 232 studies including 983,840 participants met inclusion criteria and were included in the review. Television (TV) watching was the most common measure of sedentary behaviour and body composition was the most common outcome measure. Qualitative analysis of all studies revealed a dose-response relation between increased sedentary behaviour and unfavourable health outcomes. Watching TV for more than 2 hours per day was associated with unfavourable body composition, decreased fitness, lowered scores for self-esteem and pro-social behaviour and decreased academic achievement. Meta-analysis was completed for randomized controlled studies that aimed to reduce sedentary time and reported change in body mass index (BMI) as their primary outcome. In this regard, a metaanalysis revealed an overall significant effect of -0.81 ( $95 \% \mathrm{Cl}$ of -1.44 to $-0.17, \mathrm{p}=0.01)$ indicating an overall decrease in mean BMI associated with the interventions. There is a large body of evidence from all study designs which suggests that decreasing any type of sedentary time is associated with lower health risk in youth aged 5-17 years. In particular, the evidence suggests that daily TV viewing in excess of 2 hours is associated with reduced physical and psychosocial health, and that lowering sedentary time leads to reductions in BMI.
\end{abstract}

Keywords: Inactivity, sitting, TV, body composition, fitness, metabolic syndrome, cardiovascular disease, self-esteem, pro-social behaviour, academic achievement

\section{Introduction}

Engaging in regular physical activity is widely accepted as an effective preventative measure for a variety of health risk factors across all age, gender, ethnic and socioeconomic subgroups [1-6]. However, across all age groups, levels of physical activity remain low [7-12] and obesity rates continue to rise [10,11,13,14]; collectively threatening the persistent increase in life expectancy

\footnotetext{
* Correspondence: mtremblay@cheo.on.ca

${ }^{1}$ Healthy Active Living and Obesity Research, Children's Hospital of Eastern Ontario Research Institute. 401 Smyth Road, Ottawa, Ontario, K1H 8L1, Canada

Full list of author information is available at the end of the article
}

enjoyed over the past century and efforts to counteract the inactivity and obesity crisis [15].

This inactivity crisis is especially important in the pediatric population as recent data from the Canadian Health Measures Survey [8] suggest that only 7\% of children and youth aged 6-19 years participate in at least 60 minutes of moderate- to vigorous-intensity physical activity per day, thus meeting the current physical activity guidelines from Canada [16], the U.S. [6], the U.K [17], Australia [18] and the World Health Organization (WHO) [5]. However, even for those children and youth who meet current guidelines, there remains 23 hours per day for school, sleep, work, and discretionary time. Several sources report that children and youth spend the majority of their

\section{() Biomed Central}


discretionary time engaging in sedentary pursuits (e.g. watching television (TV) or playing video games) [8,19-28]. Canadian children and youth are spending an average of 8.6 hours per day, or $62 \%$ of their waking hours being sedentary [8]. Similar trends are being reported in the U.S. where children and youth spend an average of 6-8 hours per day being sedentary [22-28]. Accumulating evidence shows that, independent of physical activity levels, sedentary behaviours are associated with increased risk of cardio-metabolic disease, all-cause mortality, and a variety of physiological and psychological problems [29-31]. Therefore, to maximize health benefits, approaches to resolve the inactivity crisis should attempt to both increase deliberate physical activity and decrease sedentary behaviours, especially in the pediatric population. However, to date, public health efforts have focused primarily on physical activity and have paid little attention to the mounting evidence to support sedentary behaviour as a distinct behaviour related to poor health.

A recent scoping review identified review articles, meta-analyses, and grey literature that examined the relationship between sedentary behaviour and health [32]. The large majority of this information reported on the relationship between screen time and body composition and did not include other indicators of health [23-25]. Furthermore, none of these reviews followed the rigorous process of a systematic review and are therefore not able to be used to inform the development of clinical practice guidelines. As a result, to our knowledge, there are no systematic, evidence-based sedentary behaviour guidelines for any age group, anywhere in the world. Guidelines that do exist are largely based on expert opinion or narrative literature reviews [33,34].

Therefore, the purpose of this systematic review was to gather, catalog, assess and evaluate the available evidence examining sedentary behaviours in relation to selected health outcomes in children and youth 5-17 years of age and present a summary of the best available evidence. Specifically, the review presents available evidence for minimal and optimal thresholds for daily sedentary time in children and youth, and when possible, how thresholds differ across health outcome or demographic status (i.e. age, gender). The information gathered in this review can serve to guide future research and inform the development of evidence-based clinical practice guideline recommendations for safe and healthy amounts of daily sedentary behaviour in the pediatric population.

\section{Methods}

\section{Study Inclusion Criteria}

The review sought to identify all studies that examined the relationship between sedentary behaviour and a specific health outcome in children and youth (aged 5-17 years).
All study designs were eligible (e.g. cross sectional, retrospective, prospective, case control, randomized controlled trial (RCT), longitudinal). Longitudinal studies were included if the data presented in the article was consistent with the age limits that were set (i.e. if the study looked at participants at age 10 and then again at age 30, only baseline measurements from age 10 were used).

Studies were included only if there was a specific measure of sedentary behaviour. Eligible exposures of sedentary behaviours included those obtained via direct (e.g., measurements of sitting, or low activity measured by accelerometer) and self-reported (e.g., questionnaires asking about TV watching, video gaming, non-school computer use, and screen time - composite measures of $\mathrm{TV}$, video games, computers) methods. Sedentary behaviour was often measured as a composite measure of all time engaging in sedentary behaviours including screen time outside of school hours. Six health indicators were chosen based on the literature, expert input, and a desire to have relevant measures from a range of holistic health indicators (i.e. not only physical health, but also emotional, mental and intellectual health). The six eligible indicators in this review were:

1. Body composition (overweight/obesity measured by body mass index (BMI), waist circumference, skin folds, bio-impedance analysis (BIA), dual-energy $x$ ray absorptiometry (DXA or DEXA));

2. Fitness (physical fitness, physical conditioning, musculoskeletal fitness, cardiovascular fitness);

3. Metabolic syndrome (MS) and cardiovascular disease (CVD) risk factors (unfavourable lipid levels, blood pressure, markers for insulin resistance or type 2 diabetes);

4. Self-esteem (self-concept, self-esteem, self efficacy);

5. Behavioural conduct/pro-social behaviour (child behaviour disorders, child development disorder, prosocial behaviour, behavioural conduct, aggression);

6. Academic achievement (school performance, grade-point average).

No Language or date limits were imposed in the search. The following definitions were used to help guide the systematic review [31]:

- Sedentary: A distinct class of behaviours (e.g. sitting, watching TV, playing video games) characterized by little physical movement and low energy expenditure ( $\leq 1.5$ METs).

- Sedentarism: Engagement in sedentary behaviours characterized by minimal movement, low energy expenditure, and rest. 
- Physically active: Meeting established physical activity guidelines (e.g. see Tremblay et al. 2011 for Canadian Physical Activity Guidelines [16]).

- Physical inactivity: The absence of physical activity, usually reflected as the proportion of time not engaged in physical activity of a pre-determined intensity and therefore not meeting established physical activity guidelines.

\section{Study Exclusion Criteria}

As the volume of literature on sedentary behaviour was anticipated to be very high, to control the feasibility of this project, the following sample size limits were set $a$ priori: population based studies (observational, cross sectional, cohort, and retrospective studies) were required to have a minimum sample size of 300 participants; RCTs, and intervention studies were required to have at least 30 participants. Studies of 'active gaming' (e.g., Nintendo Wii ${ }^{\mathrm{TM}}$, Microsoft Kinect ${ }^{\mathrm{TM}}$, Sony's Playstation Move ${ }^{\mathrm{TM}}$, video arcades, etc.) were excluded. Finally, studies that defined sedentary behaviour as 'failing to meet physical activity guidelines' were excluded from the review.

\section{Search strategy}

The following electronic bibliographic databases were searched using a comprehensive search strategy to identify relevant studies: Ovid MEDLINE(R) (1950 to February Week 2 2010), Ovid EMBASE (1980 to 2010 Week 07), and Ovid psycINFO (1806 to February Week 3 2010). The search strategy was created by a single researcher (JM) and run by a second researcher (AL). The search strategies can be found in Additional file 1. The search was limited to studies looking at 'school-aged' children and youth (mean age of 5-17 years). Articles were extracted as text files from the OVID interface and imported in to Reference Manager Software (Thompson Reuters, San Francisco, CA). Duplicate articles were first removed using Reference Manager Software, and any remaining duplicates were removed manually. All articles were given a unique reference identification number in the database.

Titles and abstracts of potentially relevant articles were screened by two reviewers (AL and one of GG, MT, RC, RL or TS) and full text copies were obtained for all articles meeting initial screening by at least one reviewer. Two independent reviewers examined all full text articles (AL and one of GG, MT, RC, RL or TS) and any discrepancies were resolved by discussion and consensus between the two reviewers. If the reviewers were unable to reach consensus, a third reviewer was asked to look at the article in question. Consensus was obtained for all included articles.
Twelve key content experts were contacted and asked to identify the most influential papers from their personal libraries examining sedentary behaviour and health in the pediatric age group. Government documents from the U.S [6], the U.K. [17], and Australia [18] were used for reference and to help guide the review process.

\section{Data extraction}

Standardized data extraction tables were created; data extraction was completed by one reviewer (AL) and checked by another (one of GG, RC, RL, or TS) for accuracy. Information was extracted regarding study characteristics (i.e. year, study design, country, number of participants, age), type of sedentary behaviour, measure of sedentary behaviour (i.e. direct, or indirect), and health outcome. Reviewers were not blinded to the authors or journals when extracting data.

\section{Risk of bias assessment}

The Downs and Black checklist was used to asses study quality [35]. This 27 point checklist assesses the quality of reporting (e.g. "Are the main findings of the study clearly described"); external validity (e.g. "Were the subjects asked to participate representative of the entire population from which they were recruited"); internal validity (e.g. "Were subjects randomized to intervention groups"); and power (e.g. "Was there sufficient power such that the difference being due to chance is less than $5 \%$ "). The maximum score a study can receive is 32 , with higher scores indicating better quality. Inter-rater reliability was calculated using Cohen's kappa.

Quality of evidence was determined by the study design and by Downs and Black score. Level of evidence was used to explain the quality of available studies and the confidence of the findings [36]. RCTs were considered to have the highest level of evidence while anecdotal reports were considered to have the lowest evidence. See Table 1 for more details. When possible, studies were examined for differences among age and gender subgroups.

\section{Analysis}

A meta-analysis was performed with the data that were sufficiently homogeneous in terms of statistical, clinical, and methodological characteristics using Review Manager Software 5.0 (The Cochrane Collaboration, Copenhagen Denmark). Pooled estimates for the meta-analysis and their $95 \%$ confidence intervals were obtained using the random effects estimator of DerSimonian-Laird [37]. Studies were weighted by the inverse of their variance. Cochrane's Q was used to test for heterogeneity among studies and the $\mathrm{I}^{2}$ (squared) index [10] was used to determine the degree of heterogeneity [38]. Funnel plots were 
Table 1 Criteria for assigning level of evidence to a recommendation

\begin{tabular}{ll}
\hline Level of evidence & \multicolumn{1}{c}{ Criteria } \\
\hline Level 1 & - Randomized control trials without important limitations \\
Level 2 & - Randomized control trials with important limitations \\
& - Observational studies (non-randomized clinical trials or cohort studies) with overwhelming evidence \\
Level 3 & - Other observational studies (prospective cohort studies, case-control studies, case series) \\
Level 4 & - Inadequate or no data in population of interest \\
& - Anecdotal evidence or clinical experience \\
\hline
\end{tabular}

Adapted from: Lau DC et al. 2007 [36]

used to assess publication bias (data not shown). Qualitative syntheses were conducted for remaining studies.

\section{Results}

Description of studies

After de-duplication, the preliminary search of electronic databases, reference lists, and grey literature identified 5,291 potentially relevant articles (Figure 1 ). Of these, 3,299 were identified in MEDLINE, 1,016 in EMBASE, 912 in psycINFO, and 64 through key informants, government documents, and bibliographies. After a preliminary review of titles and abstracts, 828 articles were included for detailed assessment of the full text article. Of these, 232 met the criteria for study inclusion (8 RCTs, 10 intervention studies, 37 longitudinal studies and 177 cross sectional studies). Individual study characteristics can be seen in Table 2. Reasons for excluding studies included: ineligible population (e.g. ineligible age or sample size) $(\mathrm{n}=161)$, ineligible exposure (e.g. diet, physical activity) $(n=145)$, ineligible measure of sedentary behaviour (i.e. not meeting physical activity guidelines) $(n=19)$, ineligible outcome $(n=60)$, ineligible analysis (e.g. analysis focused on content of screen time versus duration of screen time, analysis focused on active video gaming) $(n=60)$, and 'other' $(n=216)$ (e.g. commentary article or methodological paper). Some studies were excluded for multiple reasons. Some articles $(n=9)$ could not be retrieved due to missing or incorrect reference information.

Table 2 provides a summary of all studies included in the review. The majority of the studies included in this systematic review were cross sectional $(n=177)$. In total, data from 983,840 participants were included in this review. Studies ranged from 30 participants in intervention studies and RCTs, to 62,876 participants in cross sectional observational investigations. Articles were published over a 51 year period from 1958 to 2009, and included participants ranging from 2-19 years of age. Although the scope of the review focused on those 5-17 years of age, studies that had a range below 5 years or over 17 years were not excluded as long as the mean age was between 5-17 years. Included studies involved participants from 39 countries; there were a greater number of articles reporting on female-only data than those reporting on male-only data. Translators were contracted to read non-English articles and complete any necessary data extraction for studies that met inclusion criteria $(\mathrm{n}=8)$.

Of the 232 studies, 170 studies reported data on body composition, 15 on fitness, 11 on MS and CVD, 14 on self-esteem, 18 on pro-social behaviour, and 35 on academic achievement. The majority of studies $(n=223)$ used indirect measures to assess sedentary behaviour (i.e. parent-, teacher-, or self-report questionnaires). There were 14 studies $[24,27,28,39-49]$ that directly measured sedentary behaviour with accelerometers and one that directly measured television viewing through a monitoring device [50]. The direction of the association between increased sedentary behaviour and health outcomes were similar between direct and indirect measures. Meta-analysis was conducted for RCTs examining change in body mass index.

\section{Risk of bias assessment}

Risk of bias assessment was completed for all included studies (Additional file 2). The mean Downs and Black score was 20.7 (range $=16-26$ ). The studies were then split into groups and labeled as 'high quality' (score 23$26, \mathrm{n}=36$ ), 'moderate quality' (score 19-22, $\mathrm{n}=169$ ), and 'lower quality' (score 16-18, $\mathrm{n}=27$ ). Quality of study did not affect the outcome of the study; in other words, both lower quality and high quality studies showed a positive relationship between increased time spent sedentary and health risk. Inter-reviewer assessment using the Downs and Black tool was very high (kappa $=0.98)$.

\section{Data Synthesis \\ Body composition}

Of the 232 studies included in this review, 170 examined body composition, with the majority of these focusing on the relationship between overweight and obesity and time spent watching TV (Table 3). Body composition was measured in a variety of ways including body mass index (BMI), sum of skin folds, percent body fat and various composite measures (e.g. BMI + sum of skin folds). Of the 8 RCTs, 7 showed that decreases in sedentary time lead to reductions in body weight (see meta-analysis below for details). Intervention studies reported desirable 


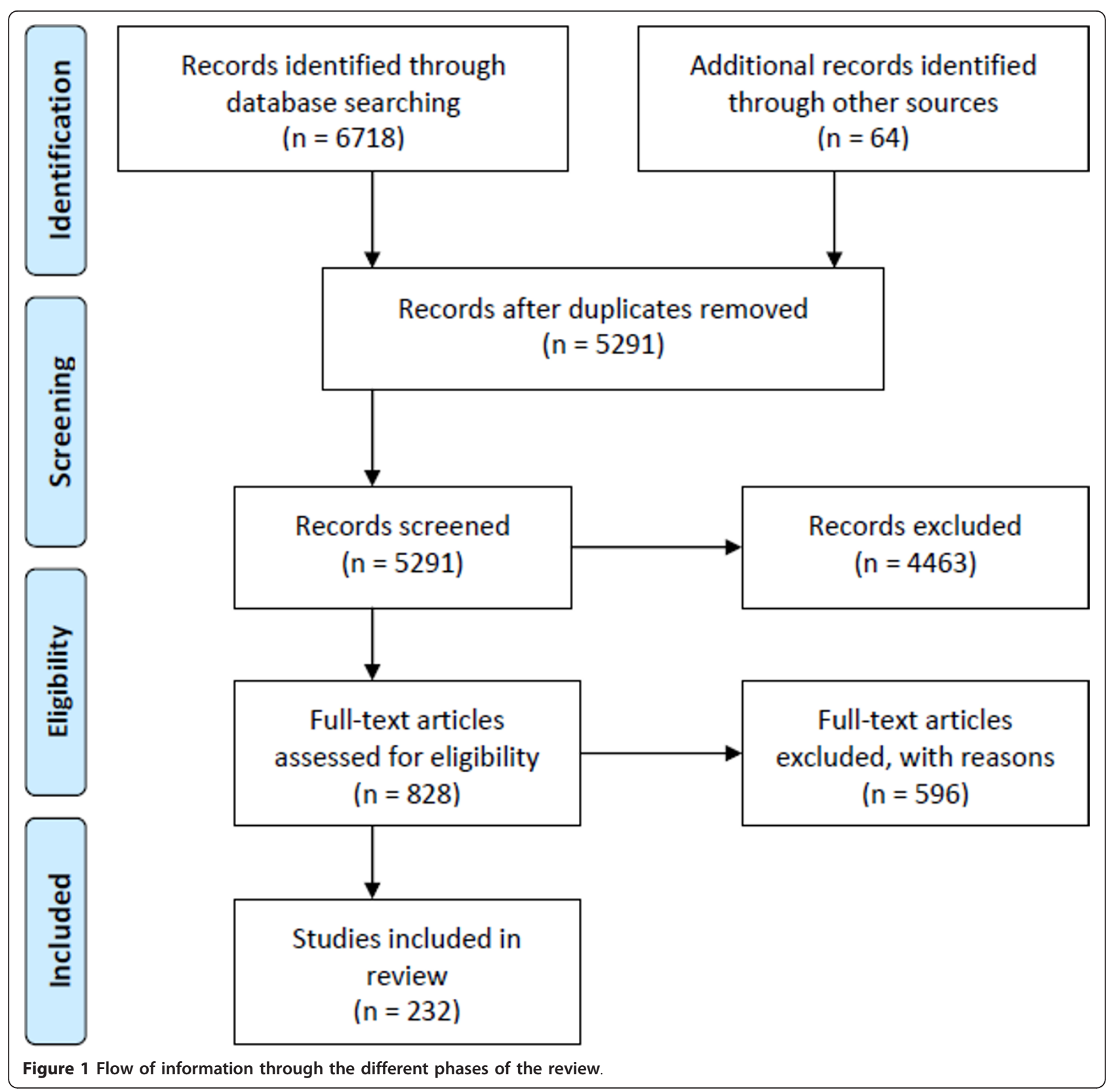

changes in body weight, BMI, and weight status among children and youth who successfully decreased their sedentary time [51-60]. Three intervention studies [61-63] reported that although sedentary behaviour decreased, there was no change in weight status (measured through BMI and skinfold thickness); however, these studies had relatively short follow-up periods ( $\sim 1$ year) and no control group leading the authors hypothesized that a longer follow up period was needed to detect a significant change in body composition. While nine-teen longitudinal studies reported that children who watched greater amounts of TV at baseline saw steeper increases in BMI, body weight and fat mass over time [64-82], nine longitudinal studies reported no significant relationship between time spent sedentary and weight status or fat mass [61-63,83-89]. Of the 119 cross sectional studies, 94 reported that increased sedentary time was associated with one or more of increased fat mass, increased BMI, increased weight status and increased risk for being overweight [28,90-182]. Risk for obesity increased in a dose response manner with increased time spent engaging in sedentary behaviours $[92,106,110,128,156,178]$. Twenty-five cross sectional studies reported no significant relationship between 


\begin{tabular}{|c|c|c|c|c|c|c|c|c|c|c|c|c|}
\hline \multirow[b]{2}{*}{ First Author } & \multirow[b]{2}{*}{ Year } & \multirow{3}{*}{ Country } & \multirow{3}{*}{ Grade } & \multirow{3}{*}{$\begin{array}{l}\text { Age } \\
\text { Range }\end{array}$} & \multirow{3}{*}{ Mean age } & \multicolumn{3}{|c|}{$n$ analyzed } & \multirow{2}{*}{\multicolumn{2}{|c|}{$\begin{array}{l}\text { Units of } \\
\text { sedentary } \\
\text { behaviour }\end{array}$}} & \multirow{3}{*}{ Exposure } & \multirow[b]{2}{*}{ Outcome } \\
\hline & & & & & & Total & Boys & Girls & & & & \\
\hline \multicolumn{8}{|c|}{$\begin{array}{l}\text { RANDOMIZED CONTROLLED } \\
\text { TRIALS }\end{array}$} & & & & & \\
\hline Epstein LH [265] & 1995 & US & & $8-12$ & 10.1 & 61 & & & hour & week & TV & BC \\
\hline Epstein LH [50] & 2008 & US & & $4-7$ & 6 & 70 & 37 & 33 & hour & day & TV & BC \\
\hline Goldfield GS [264] & 2006 & Canada & & $8-12$ & 10.4 & 30 & 13 & 17 & $\min$ & day & TV & $B C$ \\
\hline Gortmaker SL [57] & 1995 & US & & & 11.7 & 1295 & 668 & 627 & hour & day & TV & BC \\
\hline Hughes AR [262] & 1991 & Scotland & & $5-11$ & 8.8 & 134 & 59 & 74 & hour & day & SB & $B C$ \\
\hline Robinson TN [58] & 1999 & US & & & & 192 & & & hour & week & TV, GAMES & $\mathrm{BC}$ \\
\hline Robinson TN [221] & 2003 & US & & $8-10$ & 9.5 & 61 & 0 & 61 & hour & week & TV & $B C, S E$ \\
\hline Shelton D [263] & 2007 & Australia & & $3-10$ & 7.5 & 43 & 20 & 23 & hour & day & TV & $B C$ \\
\hline \multicolumn{13}{|l|}{ INTERVENTION STUDIES } \\
\hline Epstein LH [56] & 2000 & US & & $8-12$ & 10.5 & 76 & 24 & 52 & hour & month & $\mathrm{SB}, \mathrm{ST}$ & $\mathrm{BC}, \mathrm{FIT}$ \\
\hline Epstein LH [59] & 2004 & US & & $8-12$ & 9.8 & 60 & 23 & 39 & times & week & TV & BC \\
\hline Epstein LH [60] & 2005 & US & & $8-16$ & & 58 & 28 & 30 & hour & day & $\mathrm{SB}, \mathrm{TV}$ & $B C$ \\
\hline Gentile DA [61] & 2009 & US & & & 9.6 & 1323 & 685 & 674 & hour & day & ST & $B C$ \\
\hline Goldfield GS [52] & 2007 & Canada & & $8-12$ & 10.4 & 30 & 13 & 17 & hour & day & SB & $\mathrm{BC}, \mathrm{SE}$ \\
\hline Harrison M [62] & 2003 & Ireland & & & 10.2 & 312 & 177 & 135 & $\min$ & day & TV, ST & $B C$ \\
\hline Ochoa MC [53] & 2007 & Spain & & $6-18$ & 11.6 & 370 & 196 & 174 & hour & week & TV & $\mathrm{BC}$ \\
\hline Salmon J [51] & 2008 & Australia & & 1011 & 10.8 & 311 & 152 & 159 & hour & day & $T V$ & BC \\
\hline Simon C [54] & 2002 & France & & & 11.7 & 954 & 468 & 486 & hour & day & TV, COMP & $B C, S E$ \\
\hline Tanasescu M [55] & 2000 & Puerto Rico & & $7-10$ & 9.2 & 53 & 22 & 31 & hour & day & TV & BC \\
\hline LONGITUDINAL STUDIES & & & & & & & & & & hour & & \\
\hline Aires L [83] & 2010 & Portugal & & $11-19$ & & 345 & 147 & 198 & hour & day & SCREEN & $\mathrm{BC}, \mathrm{FIT}$ \\
\hline Berkey CS [76] & 2003 & US & & $10-15$ & & 11887 & 5120 & 6767 & hour & day & TV, GAMES & $B C$ \\
\hline Bhargava A [77] & 2008 & US & & & & 7635 & & & $\min$ & day & TV & $\mathrm{BC}$ \\
\hline Blair NJ [68] & 2007 & England & & & 5.5 & 591 & 287 & 304 & hour & day & $\mathrm{SB}, \mathrm{TV}$ & $B C$ \\
\hline Borradaile KE [86] & 2008 & US & & & 11.2 & 1092 & 501 & 591 & hour & week & TV & $B C$ \\
\hline Burke V [71] & 2006 & Australia & & & $7.6 / 10.8$ & 1569 & 630 & 648 & hour & week & SCREEN & $B C$ \\
\hline Chen JL [78] & 2007 & Chinese & & $7-8$ & 7.52 & 307 & 147 & 160 & hour & day & TV, GAMES & $B C$ \\
\hline Danner FW [66] & 2008 & US & & & & 7334 & 3674 & 3660 & hour & day & TV & $B C$ \\
\hline Dasgupta K [215] & 2006 & Canada & & & $\begin{array}{l}12.7 / 15.1 / \\
17.0\end{array}$ & 662 & 319 & 343 & hour & week & $\mathrm{SB}, \mathrm{TV}$ & MS \\
\hline Day RS [85] & 2009 & US & & $8-14$ & & 556 & 277 & 279 & $\min$ & day & TV & $B C$ \\
\hline Dietz WH [181] & 1985 & US & & $12-17$ & & 2153 & & & hour & day & TV & $B C$ \\
\hline Elgar FJ [79] & 2005 & Wales & & & 11.7 & 654 & 293 & 361 & hour & week & TV & $B C$ \\
\hline Elgar FJ [79] & 2005 & Wales & & & 15.3 & 392 & 181 & 211 & hour & week & TV & $B C$ \\
\hline Ennemoser M [237] & 2007 & German & & $6-8$ & & 332 & & & $\min$ & day & $\mathrm{TV}$ & $\mathrm{SE}, \mathrm{AA}$ \\
\hline Fulton JE [84] & 2009 & US & & $10-18$ & & 472 & 245 & 227 & $\min$ & day & TV & $\mathrm{BC}$ \\
\hline Gable S [70] & 2007 & US & & & & 8000 & & & hour & day & TV & $B C$ \\
\hline Hancox RJ [88] & 2004 & New Zealand & & $5-15$ & & 1013 & & & hour & day & TV & $\mathrm{BC}, \mathrm{MS}$ \\
\hline Hancox RJ [72] & 2006 & New Zealand & & $5-15$ & & 603 & 372 & 339 & hour & day & SCREEN & BC \\
\hline Henderson VR [67] & 2007 & US & & $11-19$ & & 2379 & 0 & 2379 & hour & day & TV, SCREEN & $B C$ \\
\hline Hesketh K [80] & 1997 & Australia & & $5-10$ & 7.6 & 1278 & 630 & 648 & hour & day & SCREEN & $B C$ \\
\hline Hesketh K [80] & 1997 & Australia & & $8-13$ & 10.7 & 1278 & 630 & 648 & hour & day & SCREEN & $B C$ \\
\hline Hesketh K [64] & 2009 & Australia & & $5-10$ & 7.7 & 1943 & 972 & 971 & hour & day & TV, GAMES & $\mathrm{BC}$ \\
\hline Hesketh K [64] & 2009 & Australia & & $8-13$ & & 1569 & 816 & 753 & hour & day & TV, GAMES & $B C$ \\
\hline Jackson LA [223] & 2009 & US & & & 12 & 500 & 235 & 265 & hour & day & $\begin{array}{l}\text { COMP, } \\
\text { SCREEP }\end{array}$ & SE \\
\hline Jago R [82] & 2005 & US & & $5-6$ & 6.5 & 138 & 65 & 73 & $\min$ & $\mathrm{hr}$ & $\mathrm{SB}, \mathrm{TV}$ & $B C$ \\
\hline Janz KF [73] & 2005 & US & & & $5.6 / 8.6$ & 378 & 176 & 202 & hour & day & SCREEN & $B C$ \\
\hline Johnson JG [41] & 2007 & US & & & & & & & hour & day & $\mathrm{TV}$ & $\mathrm{AA}$ \\
\hline
\end{tabular}


Table 2 Summary of characteristics of included studies (Continued)

\begin{tabular}{|c|c|c|c|c|c|c|c|c|c|c|c|c|}
\hline Kaur H [75] & 2003 & US & & $12-17$ & & 2223 & 1149 & 1074 & hour & day & TV & $B C$ \\
\hline Lajunen HR [128] & 2007 & Finland & & $15-19$ & & 5184 & & & hour & & SB & $B C$ \\
\hline Lonner W [238] & 1985 & US & & $9-19$ & 14.2 & 367 & & & hour & day & $T V$ & $\mathrm{AA}$ \\
\hline Maffeis C [89] & 1998 & Italy & & & 8.7 & 298 & 148 & 150 & $\min$ & day & SCREEN & $\mathrm{BC}$ \\
\hline Mistry K [229] & 2007 & US & & & & & & & hour & day & $\mathrm{TV}$ & $\mathrm{PRO}$ \\
\hline Mitchell JA [49] & 2009 & UK & & $11-12$ & 11.8 & 5434 & 2590 & 2844 & hour & day & SB & $\mathrm{BC}, \mathrm{FIT}$ \\
\hline Must A [87] & 2007 & US & & $10-17$ & & 156 & 0 & 156 & hour & day & SB, SCREEN & $B C$ \\
\hline O'Brien M [69] & 2007 & US & & $2-12$ & & 653 & & & hour & week & $T V$ & $B C$ \\
\hline Parsons TJ [74] & 2005 & England/Scot & land/Wal & & $11 / 16$ & 17733 & & & hour & day & TV & $\mathrm{BC}$ \\
\hline Purslow LR [63] & 2008 & England & & 8-9 & & 345 & 176 & 169 & $\min$ & day & SB & $B C$ \\
\hline Timperio A [65] & 2008 & Australia & & $10-12$ & & 344 & 152 & 192 & times & week & SB, SCREEN & $B C$ \\
\hline Treuth MS [29] & 2007 & US & & & 11.9 & 984 & 0 & 984 & $\min$ & day & SB & $B C$ \\
\hline Treuth MS [27] & 2009 & US & & & 13.9 & 984 & 0 & 984 & $\min$ & day & SB & $B C$ \\
\hline Wosje,K.S [205] & 2009 & US & & $6.75-7.25$ & & 214 & & & hour & day & SCREEN & FIT \\
\hline \multicolumn{13}{|l|}{ CROSS SECTIONAL STUDIES } \\
\hline Al SH [192] & 2009 & International & & $12-18$ & & 17715 & 8503 & 9212 & hour & day & TV & $\mathrm{BC}$ \\
\hline Albarwani S [207] & 2009 & Oman & & $15-16$ & & 529 & 245 & 284 & hour & week & TV, COMP & FIT \\
\hline Alves JG [191] & 2009 & Brazil & & $7-10$ & & 733 & 407 & 326 & hour & day & TV & $\mathrm{BC}$ \\
\hline Aman J [218] & 2009 & Sweden & & $11-18$ & 14.5 & 2093 & 1016 & 991 & hour & week & TV, COMP & MS \\
\hline Andersen LF [155] & 2005 & Norway & & 8-14 & & 1432 & 702 & 730 & hour & day & TV & $\mathrm{BC}$ \\
\hline Andersen RE [142] & 1998 & US & & 8-16 & & 4063 & 1985 & 2071 & hour & day & $T \mathrm{~V}$ & $B C$ \\
\hline Anderson SE [103] & 2008 & US & & $4-12$ & 8 & 2964 & 1509 & 1455 & hour & day & TV & $\mathrm{BC}$ \\
\hline Armstrong CA [213] & 1998 & US & & & 9.28 & 588 & 304 & 284 & hour & day & $T \mathrm{~V}$ & FIT \\
\hline Asante PA [183] & 2009 & US & & $3-13$ & 8.5 & 324 & 182 & 142 & hour & day & SCREEN & $\mathrm{BC}$ \\
\hline Aucote HM [163] & 2009 & Australia & $5-6$ & & 11.09 & 393 & 198 & 195 & hour & week & TV, GAMES & $\mathrm{BC}$ \\
\hline Barlow SE [151] & 2007 & US & & $6-17$ & 12.1 & 52845 & & & hour & day & TV & $B C$ \\
\hline Basaldua N [109] & 2008 & Mexico & & $6-12$ & 8.9 & 551 & 278 & 273 & hour & day & TV & $\mathrm{BC}$ \\
\hline Bellisle F [123] & 2007 & France & & $9-11$ & & 1000 & 500 & 500 & hour & day & TV & $\mathrm{BC}$ \\
\hline Berkey CS [90] & 2000 & US & & & Sep-14 & 10769 & 4620 & 6149 & hour & day & TV & $\mathrm{BC}$ \\
\hline Beyerlein A [105] & 2008 & Germany & & $4.5-7.3$ & & 4967 & 2585 & 2382 & hour & day & TV & $\mathrm{BC}$ \\
\hline Boone JE [164] & 2007 & US & & & 15.9 & 9155 & 4879 & 4276 & hour & week & SCREEN & $\mathrm{BC}$ \\
\hline Boone-Heinonen J [104] & 2008 & US & & $11-21$ & & 9251 & & & hour & & SB & $\mathrm{BC}$ \\
\hline Boutelle KN [130] & 2007 & US & & $16-18$ & & 1726 & 890 & 836 & hour & day & TV & $B C$ \\
\hline Brodersen NH [235] & 2005 & England & & & 11.8 & 4320 & 2578 & 1742 & hour & week & SB & $\mathrm{SE}, \mathrm{PRO}$ \\
\hline $\begin{array}{l}\text { Bukara-Radujkovic G } \\
\text { [96] }\end{array}$ & 2009 & Bosnia & & $11-12$ & 11.5 & 1204 & 578 & 626 & hour & day & TV, COMP & $\mathrm{BC}$ \\
\hline Butte NF [119] & 2007 & US & & $6-17$ & 10.8 & 897 & 441 & 456 & hour & day & SCREEN & $B C$ \\
\hline Caldas S [245] & 1999 & US & & $4-19$ & & 34542 & & & hour & day & TV & $\mathrm{AA}$ \\
\hline Carvalhal MM [131] & 2007 & Portugal & $10-11$ & & & 3365 & 1755 & 1610 & hour & day & TV, COMP & $\mathrm{BC}$ \\
\hline Chaput J [154] & 2006 & Canada & & $5-10$ & 6.6 & 422 & 211 & 211 & hour & day & SCREEN & $\mathrm{BC}$ \\
\hline Chen MY [78] & 2007 & Taiwan & & $13-18$ & 15.03 & 660 & 351 & 309 & hour & day & TV, COMP & $\begin{array}{l}\mathrm{BC}, \mathrm{SE} \\
\mathrm{PRO}\end{array}$ \\
\hline Chowhan J [232] & 2007 & Canada & & $12-15$ & & 2666 & & & hour & day & TV & PRO \\
\hline Christoforidis A [95] & 2009 & Greece & & $4-18$ & 11.41 & 1549 & 735 & 814 & hour & day & SCREEN & $\mathrm{BC}, \mathrm{FIT}$ \\
\hline Collins AE [149] & 2008 & Indonesia & & $12-15$ & & 1758 & 815 & 916 & hour & day & TV, COMP & $\mathrm{BC}$ \\
\hline Colwell J [200] & 2003 & Japan & & $12-13$ & & 305 & 159 & 146 & hour & day & SCREEN & $\mathrm{BC}, \mathrm{PRO}$ \\
\hline Cooper H [247] & 1999 & US & $7-11$ & & & 424 & 225 & 199 & hour & day & $T \mathrm{~V}$ & $\mathrm{AA}$ \\
\hline Crespo CJ [177] & 2001 & US & & $8-16$ & & 4069 & 1994 & 2075 & hour & day & TV & $B C$ \\
\hline Da CR [157] & 2003 & Brazil & & $7-10$ & & 446 & 107 & 107 & hour & day & $T V$ & $B C$ \\
\hline Dasgupta K [215] & 2007 & Canada & & $13-17$ & & 1267 & & & hour & week & SCREEN & MS \\
\hline Delva J [125] & 2007 & US & & & & 11265 & 5274 & 5991 & hour & week & $T V$ & $B C$ \\
\hline Dietz WH [181] & 1985 & US & & $12-17$ & & 6671 & & & hour & day & TV & $\mathrm{AA}$ \\
\hline Dietz WH [181] & 1985 & US & & $6-11$ & & 6965 & & & hour & day & $\mathrm{TV}$ & $\mathrm{BC}, \mathrm{AA}$ \\
\hline Dollman J [211] & 2006 & Australia & 6 & $10-11$ & & 843 & 439 & 404 & $\min$ & Day & $T V$ & FIT \\
\hline
\end{tabular}


Table 2 Summary of characteristics of included studies (Continued)

\begin{tabular}{|c|c|c|c|c|c|c|c|c|c|c|c|c|}
\hline Dumais SA [255] & 2009 & US & & $10-12$ & & 15850 & & & hour & & TV & AA \\
\hline Dominick JR [225] & 1984 & US & 10,11 & $14-18$ & & 250 & 110 & 140 & hour & Day & TV, GAME & SE, PRO \\
\hline Eisenmann JC [175] & 2002 & US & & $14-18$ & & 15143 & & & hour & day & TV & $\mathrm{BC}$ \\
\hline Ekelund U [134] & 2006 & Europe & & $9-16$ & & 1921 & 911 & 1010 & hour & day & TV & $\mathrm{BC}, \mathrm{MS}$ \\
\hline Fetler M [249] & 1984 & US & 6 & & & 10603 & & & hour & day & SCREEN & $\mathrm{AA}$ \\
\hline Forshee RA [201] & 2004 & US & & $12-16$ & 14 & 2216 & 1075 & 1141 & hour & day & TV & $\mathrm{BC}$ \\
\hline Forshee RA [188] & 2009 & US & & $5-18$ & & 1459 & 734 & 725 & hour & week & SCREEN & $\mathrm{BC}$ \\
\hline Gaddy GD [257] & 1986 & US & & & & 5074 & & & hour & day & TV & AA \\
\hline Giammattei J [140] & 2003 & US & & $11-14$ & 12.6 & 385 & 186 & 199 & hour & day & TV & $B C$ \\
\hline Gibson S [156] & 2004 & England & & $7-18$ & & 1294 & 655 & 639 & $\min$ & day & TV & $\mathrm{BC}$ \\
\hline Gomez LF [150] & 2007 & Colombia & & $5-12$ & & 11137 & 5539 & 5598 & hour & day & TV, GAMES & $\mathrm{BC}$ \\
\hline Gordon-Larsen P [176] & 2002 & US & & $11-19$ & 15.9 & 12759 & 6290 & 6496 & hour & week & TV, GAMES & $\mathrm{BC}$ \\
\hline Gortmaker SL [57] & 1999 & US & & $6-11$ & & 1745 & & & $\min$ & week & TV & SE, AA \\
\hline Gortmaker SL [57] & 1999 & US & & $12-17$ & & 1745 & & & $\min$ & week & TV & $\mathrm{SE}, \mathrm{AA}$ \\
\hline Graf C [167] & 2004 & Germany & & & 6.8 & 344 & 177 & 167 & hour & day & TV, COMP & $\mathrm{BC}$ \\
\hline Grusser SM [40] & 2005 & Germany & 6 & & 11.83 & 323 & 175 & 148 & hour & day & TV & $\mathrm{AA}$ \\
\hline Hardy LL [133] & 2006 & Australia & & $11-15$ & & 2750 & 1446 & 1304 & hour & day & SCREEN & FIT \\
\hline Hernandez B [178] & 1999 & Mexico & & $9-16$ & & 461 & 244 & 217 & hour & day & TV & $\mathrm{BC}$ \\
\hline Hirschler V [144] & 2009 & Argentina & & $7-11$ & 8.9 & 330 & 168 & 162 & hour & day & TV & $B C$ \\
\hline Holder MD [222] & 2009 & Canada & & $8-12$ & & 375 & 252 & 262 & hour & day & SCREEN & SE \\
\hline Hume C [190] & 2009 & Netherlands & & & 13 & 580 & 277 & 303 & hour & day & SCREEN & $\mathrm{BC}$ \\
\hline Islam-Zwart K [195] & 2008 & US & & & & 480 & 198 & 282 & hour & day & TV & $\mathrm{BC}$ \\
\hline Jackson LA [223] & 2009 & US & & & 12.18 & 515 & 259 & 256 & hour & day & $\begin{array}{l}\text { GAMES, } \\
\text { COMP }\end{array}$ & AA \\
\hline Jaruratanasirikul S [241] & 2009 & Thailand & $7-12$ & & 15.9 & 1492 & 562 & 929 & hour & & GAMES & AA \\
\hline Johnson CC [41] & 2007 & US & & & 12 & 1397 & 0 & 1397 & hour & day & SB & SE \\
\hline Katzmarzyk PT [197] & 1998 & Canada & & $9-18$ & & 784 & 423 & 361 & $\min$ & day & $\mathrm{TV}$ & $\mathrm{BC}, \mathrm{FIT}$ \\
\hline Katzmarzyk PT [184] & 1998 & Canada & & & & 640 & 356 & 284 & hour & day & TV & $\mathrm{BC}, \mathrm{FIT}$ \\
\hline Kautiainen S [135] & 2005 & Finland & & $14-18$ & & 6515 & 2916 & 3599 & hour & day & SCREEN & $\mathrm{BC}$ \\
\hline Keith TZ [256] & 1986 & US & high sC & hool seniors & & 28051 & & & hour & day & TV & AA \\
\hline Klein-Platat C [165] & 2005 & France & & & 12 & 2714 & 1357 & 1357 & hour & week & SB & $B C$ \\
\hline Kosti RI [196] & 2007 & Greece & & $12-17$ & & 2008 & 1021 & 987 & hour & day & TV & $\mathrm{BC}$ \\
\hline Kristjansson AL [243] & 2009 & Iceland & & $14-15$ & & 5810 & 2807 & 3004 & hour & day & TV & AA \\
\hline Kuntsche E [230] & 2006 & International & & $11-15$ & & 31177 & & & hour & day & TV & PRO \\
\hline Kuriyan R [117] & 2007 & India & & $6-16$ & & 598 & 324 & 274 & hour & day & TV & $\mathrm{BC}$ \\
\hline Lagiou A [160] & 2008 & Greece & & $10-12$ & & 633 & 316 & 317 & hour & day & TV, GAMES & $B C$ \\
\hline Lajous M [92] & 2009 & Mexico & & $11-18$ & 13.9 & 9132 & 3519 & 5613 & hour & day & TV & $\mathrm{BC}$ \\
\hline Lajunen HR [128] & 2007 & Finland & & & 17.6 & 4098 & 1981 & 2117 & hour & week & COMP & $\mathrm{BC}$ \\
\hline Lasserre AM [116] & 2007 & Switzerland & & $10.1-14.9$ & 12.3 & 5207 & 2621 & 2586 & hour & day & TV & $\mathrm{BC}$ \\
\hline Laurson KR [107] & 2008 & US & & $7-12$ & & 709 & 318 & 391 & hour & week & SCREEN & $\mathrm{BC}$ \\
\hline Lazarou C [217] & 2009 & Cyprus & & & 11.7 & 622 & 306 & 316 & hour & day & TV & MS \\
\hline Leatherdale ST [11] & 2008 & Canada & & $14-19$ & & 25416 & 12806 & 12610 & hour & day & TV & $\mathrm{BC}, \mathrm{PRO}$ \\
\hline Lioret S [127] & 2007 & France & & $3-14$ & & 1016 & 528 & 488 & hour & day & $\mathrm{SB}, \mathrm{TV}, \mathrm{COMP}$ & $\mathrm{BC}$ \\
\hline Lobelo F [208] & 2009 & US & & $14-18$ & & 5210 & 0 & 5210 & hour & day & SCREEN & $\mathrm{FIT}$ \\
\hline Lowry R [173] & 2002 & US & & & & 15349 & 7445 & 7828 & hour & day & TV & $\mathrm{BC}$ \\
\hline Lutfiyya MN [118] & 2007 & US & & $5-17$ & & 7972 & & & hour & day & TV & $\mathrm{BC}$ \\
\hline Maffeis C [114] & 2008 & Italy & & 8-10 & 9.3 & 1837 & 924 & 913 & hour & day & TV & $\mathrm{BC}$ \\
\hline Mark AE [220] & 2008 & US & & $12-19$ & 15.9 & 1803 & 1005 & 798 & hour & day & $\mathrm{TV}$ & $\mathrm{BC}, \mathrm{MS}$ \\
\hline McMurray RG [187] & 2000 & US & & $10-16$ & 12.7 & 2389 & 1149 & 1240 & hour & day & TV & $\mathrm{BC}$ \\
\hline
\end{tabular}


Table 2 Summary of characteristics of included studies (Continued)

\begin{tabular}{|c|c|c|c|c|c|c|c|c|c|c|c|c|}
\hline Mikolajczyk RT [194] & 2008 & Germany & & $11-17$ & 13.5 & 4878 & 2433 & 2445 & hour & $\begin{array}{l}\text { low/ } \\
\text { high }\end{array}$ & SB & $B C$ \\
\hline Moraes SA [135] & 2006 & Mexico & & $6-14$ & $8.0 / 11.3$ & 662 & 343 & 339 & hour & week & & \\
\hline Morgenstern M [94] & 2009 & Germany/US & & $10-17$ & 12.8 & 4810 & 2294 & 2516 & hour & day & SCREEN & $B C$ \\
\hline Morgenstern M [94] & 2009 & Germany/US & & $12-16$ & 14 & 4473 & 2239 & 2234 & hour & day & SCREEN & BC \\
\hline Mota J [199] & 2006 & Portugal & & & 14.6 & 450 & 220 & 230 & hour & day & TV, COMP & $B C$ \\
\hline Muller MJ [179] & 1999 & Germany & & $5-7$ & & 1468 & 739 & 729 & hour & day & TV & $B C$ \\
\hline Nagel G [193] & 2009 & Germany & & $6-9$ & 7.57 & 1079 & & 498 & hour & day & TV, GAMES & $B C$ \\
\hline $\begin{array}{l}\text { nastassea-Vlachou K } \\
\text { [240] }\end{array}$ & 1996 & Greece & & $6-13$ & & 4690 & 2279 & 2411 & hour & day & TV & AA \\
\hline Nawal LM [148] & 1998 & US & & $5-18$ & & 62976 & & & hour & day & TV, COMP & $B C$ \\
\hline Nelson MC [233] & 2006 & US & & $7-12$ & & 11957 & 5979 & 5978 & hour & day & SCREEN & PRO \\
\hline $\begin{array}{l}\text { Neumark-Sztainer D } \\
{[224]}\end{array}$ & 2004 & US & & $11-18$ & 14.9 & 4746 & 2382 & 2364 & hour & week & TV & $\mathrm{SE}, \mathrm{PRO}$ \\
\hline Nogueira JA [45] & 2009 & Brazil & & $8.3-16.8$ & 13 & 326 & 204 & 122 & hour & day & SB & $B C$ \\
\hline Obarzanek E [180] & 1994 & US & & $9-10$ & 10.1 & 2379 & 0 & 2379 & hour & week & TV & BC \\
\hline Ohannessian CM [226] & 2009 & US & & $14-16$ & 14.99 & 328 & 138 & 190 & hour & day & SCREEN & $\begin{array}{l}\text { SE, PRO, } \\
\text { AA }\end{array}$ \\
\hline Ortega FB [122] & 2007 & Spain & & $13-18.5$ & 15.4 & 2859 & 1357 & 1502 & hour & day & SB & $B C$ \\
\hline Overby NC [219] & 2009 & Norway & & $6-19$ & & 723 & 375 & 348 & $\min$ & day & TV & \\
\hline Ozmert E [42] & 2002 & Turkey & & & & 689 & 343 & 346 & hour & day & TV & PRO, AA \\
\hline Padez C [99] & 2009 & Portugal & & $7-9$ & & 3390 & 1696 & 1694 & hour & day & TV & $B C$ \\
\hline Page RM [234] & 2001 & Philippine & & & 15.1 & 3307 & 1267 & 1819 & hour & week & TV & PRO \\
\hline Pate RR [210] & 2006 & US & & $12-19$ & 15.4 & 3287 & 1686 & 1601 & hour & day & TV & FIT \\
\hline Patrick K [169] & 2004 & US & & $11-15$ & 12.7 & 878 & 407 & 471 & $\min$ & day & $\mathrm{TV}$ & $B C$ \\
\hline Pratt C [101] & 2008 & US & & & 12 & 1458 & 223 & 1235 & hour & day & SB & $B C$ \\
\hline Purath J [185] & 1995 & US & $3-5$ & & & 365 & 189 & 176 & hour & day & TV & $\mathrm{BC}, \mathrm{MS}$ \\
\hline Ramos E [126] & 2007 & Portugal & & 13 & & 2161 & 1045 & 1116 & $\min$ & week & $\mathrm{SB}, \mathrm{TV}, \mathrm{COMP}$ & $B C$ \\
\hline Rapp K [138] & 2005 & Germany & & & 6.2 & 2140 & 1015 & 1125 & hour & day & TV & $B C$ \\
\hline Ridley-Johnson R [252] & 1983 & US & $5-8$ & & & 290 & & & hour & day & TV & AA \\
\hline Roberts DF [250] & 1984 & US & & & & 539 & & & hour & week & TV & AA \\
\hline Robinson TN [58] & 1999 & US & & & 12.4 & 971 & 0 & 971 & hour & day & TV & $B C$ \\
\hline $\begin{array}{l}\text { Ruangdaraganon N } \\
\text { [141] }\end{array}$ & 2002 & Thailand & & $6-12$ & 9.4 & 4197 & 2126 & 2035 & hour & day & TV & BC \\
\hline Russ SA [147] & 2009 & US & & $6-17$ & & 54863 & 28153 & 26710 & hour & day & SCREEN & $\mathrm{BC}, \mathrm{SE}$ \\
\hline Sakamoto A [236] & 1994 & Japan & $4-6$ & & & 307 & 165 & 142 & times & week & GAMES & PRO \\
\hline Sakamoto A [236] & 1994 & Japan & $4-6$ & & & 537 & 287 & 250 & hour & week & $\begin{array}{l}\text { COMP, } \\
\text { GAMES }\end{array}$ & PRO \\
\hline Sakamoto A [236] & 1994 & Japan & $4-5$ & & & 118 & 118 & 0 & hour & week & $\begin{array}{l}\text { COMP, } \\
\text { GAMES }\end{array}$ & PRO \\
\hline Salmon J [136] & 2006 & Australia & & $5-12$ & & 1560 & 743 & 817 & hour & day & TV & $B C$ \\
\hline Sardinha LB [48] & 2008 & Portugal & & $9-10$ & 9.8 & 308 & 161 & 147 & hour & day & SB & MS \\
\hline Scott LF [254] & 1958 & US & $6-7$ & & & 407 & & & hour & & TV & AA \\
\hline Sharif I [244] & 2006 & US & & $10-14$ & & 6522 & 3169 & 3353 & hour & day & TV, GAMES & PRO, AA \\
\hline Sharif I [260] & 2010 & US & & $9-15$ & 12 & 4508 & 2209 & 2299 & hour & day & TV, GAMES & AA \\
\hline Shejwal B [246] & 2006 & India & & & 16.05 & 654 & 368 & 286 & hour & day & TV & AA \\
\hline Shields M [162] & 2006 & US/Can & & $2-17$ & & 8661 & & & hour & day & $\mathrm{SB}, \mathrm{TV}$ & $B C$ \\
\hline Shin N [239] & 2004 & US & & $6-13$ & 9 & 1203 & 605 & 598 & $\min$ & day & TV & AA \\
\hline Singh GK [106] & 2003 & US & & $10-17$ & & 46707 & 24072 & 22635 & hour & day & $T V$ & $B C$ \\
\hline Singh GK [106] & 2003 & US & & $10-17$ & & 46707 & 24072 & 22635 & hour & day & TV & $B C$ \\
\hline Skoric MM [258] & 2009 & Singapore & & $8-12$ & 10 & 333 & 180 & 153 & hour & & TV, GAMES & AA \\
\hline Smith BJ [161] & 2007 & Fiji & & $11-16$ & & 443 & 200 & 245 & hour & day & TV & $B C$ \\
\hline Spinks AB [124] & 2007 & Australia & & $5-12$ & & 518 & 282 & 236 & $\min$ & week & SB, SCREEN & $B C$ \\
\hline Steffen LM [98] & 2009 & US & & 8-11 & & 526 & 256 & 270 & hour & day & TV & $B C$ \\
\hline Stettler N [168] & 2004 & Switzerland & & & 8 & 872 & 410 & 462 & hour & day & TV, GAMES & $B C$ \\
\hline
\end{tabular}


Table 2 Summary of characteristics of included studies (Continued)

\begin{tabular}{|c|c|c|c|c|c|c|c|c|c|c|c|c|}
\hline Sugiyama T [47] & 2007 & US & & $12-19$ & 15.9 & 4508 & 2295 & 2213 & hour & day & SB & MS \\
\hline Sun $Y[91]$ & 2009 & Japan & & $12-13$ & & 5753 & 2842 & 2911 & hour & day & TV & $B C$ \\
\hline Taylor WC [158] & 2002 & US & & $6-15$ & 11.1 & 509 & 231 & 278 & kcal & day & SB & $B C$ \\
\hline te Velde SJ [129] & 2007 & International & & $9-14$ & 11.4 & 12538 & 6256 & 6282 & hour & day & TV, COMP & $B C$ \\
\hline Thompson AM [189] & 2009 & Canada & $\begin{array}{l}3,7 \\
11\end{array}$ & & & 1777 & 795 & 982 & $\min$ & day & TV & $B C$ \\
\hline Toschke AM [112] & 2008 & Germany & & $5-6$ & & 4884 & & & hour & day & TV & $B C$ \\
\hline Toschke AM [121] & 2007 & Germany & & $5-6$ & & 5472 & & & hour & day & TV & $B C$ \\
\hline Trang NHHD [146] & 2009 & Australia & & $11-16$ & & 2660 & 1332 & 1328 & hour & day & SCREEN & $B C$ \\
\hline Tremblay MS [172] & 2003 & Canada & & $7-11$ & & 7261 & & & hour & day & TV & $\mathrm{BC}$ \\
\hline Treuth MS [27] & 2009 & US & & $11-12$ & 11.9 & 1579 & 0 & 1579 & hour & day & SB & $B C$ \\
\hline Tsai H [153] & 2007 & Taiwan & & $11-12$ & & 2218 & 1146 & 1072 & hour & day & TV & $\mathrm{BC}$ \\
\hline Tsai H [145] & 2009 & Taiwan & & $11-12$ & & 1329 & 615 & 672 & hour & day & $\mathrm{SB}, \mathrm{TV}$ & $B C$ \\
\hline Tucker LA [212] & 1987 & US & & & 15.7 & 406 & 406 & 0 & hour & day & TV & $\begin{array}{l}\text { FIT, SE, } \\
\text { PRO }\end{array}$ \\
\hline Tucker LA [206] & 1986 & US & & & 15.7 & 379 & 379 & 0 & hour & day & TV & FIT \\
\hline Tucker LA [214] & 1996 & US & & $9-10$ & 9.8 & 262 & 162 & 100 & hour & day & TV & FIT \\
\hline Ussher MH [231] & 1007 & England & & $13-16$ & & 2623 & & & hour & day & TV & $\mathrm{PRO}, \mathrm{AA}$ \\
\hline Utter J [171] & 2003 & US & & & 14.9 & 4480 & 2240 & 2240 & hour & day & SCREEN & $B C$ \\
\hline Utter J [152] & 2007 & New Zealand & & $5-14$ & & 1743 & 959 & 784 & hour & day & TV, COMP & $B C$ \\
\hline Vader AM [97] & 2009 & US & & & 11,7 & 11594 & 6162 & 5432 & hour & day & TV & $B C$ \\
\hline van Schie EG [261] & 1997 & Netherlands & & $10-14$ & 11.5 & 346 & 171 & 175 & hour & day & SCREEN & $\mathrm{PRO}, \mathrm{AA}$ \\
\hline van Zutphen M [159] & 2007 & Australia & & $4-12$ & 8 & 1926 & 939 & 987 & $\min$ & day & TV & $\mathrm{BC}$ \\
\hline Vandewater EA [170] & 2004 & US & & $1-12$ & 6 & 2831 & 1444 & 1387 & hour & day & SB, SCREEN & $B C$ \\
\hline Vaughan C [198] & 2007 & Australia & & $11-18$ & 14 & 443 & 189 & 254 & hour & day & SCREEN & $B C$ \\
\hline $\begin{array}{l}\text { Vicente-Rodriguez G } \\
\text { [110] }\end{array}$ & 2008 & Spain & & $13-18.5$ & & 1960 & 1012 & 948 & hour & day & TV, GAMES & $B C$ \\
\hline Violante R [137] & 2005 & Mexico & & $6-14$ & & 8624 & 258 & 4366 & hour & day & TV & $B C$ \\
\hline Wake M [186] & 2003 & Australia & & $5-13$ & 9.1 & 2862 & 1445 & 1417 & hour & week & SCREEN & $B C$ \\
\hline Walberg HJ [251] & 1984 & US & $2-6$ & & 13 & 2890 & 1445 & 1445 & hour & day & TV & AA \\
\hline Walberg HJ [253] & 1982 & US & & & 17 & 2001 & 1031 & 970 & hour & day & TV & AA \\
\hline Waller CE [202] & 2003 & China & & $6-11$ & 9 & 880 & & & hour & week & TV & $B C$ \\
\hline Wang Y [120] & 2007 & US & & & 11.9 & 498 & 218 & 280 & hour & day & SCREEN & $B C$ \\
\hline Welch WW [248] & 1986 & Australia & $3-4$ & 9 & 9 & 1960 & & & & & TV & AA \\
\hline Wells JC [108] & 2008 & Brazil & & $10-12$ & & 4452 & 2193 & 2258 & hour & day & TV & $\mathrm{BC}, \mathrm{MS}$ \\
\hline Whitt-Glover MC [24] & 2009 & US & & $6-19$ & & 749 & 351 & 398 & $\min$ & day & SB & $\mathrm{BC}$ \\
\hline Wiggins J [227] & 1987 & US & $4-12$ & & & 483 & 252 & 231 & $\min$ & day & TV & $\mathrm{SE}, \mathrm{AA}$ \\
\hline Wolf AM [203] & 1998 & US & & $11-14$ & & 552 & 0 & 552 & hour & day & TV & $B C$ \\
\hline Wong SL [100] & 2009 & Canada & & & 15.5 & 25060 & 12806 & 12254 & hour & day & SB, SCREEN & $B C$ \\
\hline Zabinski MF [132] & 2007 & US & & $11-15$ & & 878 & 425 & 453 & hour & day & SB & $B C$ \\
\hline
\end{tabular}

SB, sedentary behaviour; TV, television viewing; COMP, computer time; GAME, video game playing; SCREEN, composite measure of 2 or more screen activities (i.e. television viewing, computer time, or video game playing); BC, body composition; MS, measures of metabolic syndrome and/or cardiovascular disease (e.g. insulin resistance, blood pressure); $\mathrm{SE}$, self-esteem; $\mathrm{PRO}$, pro-social behaviour; $\mathrm{AA}$, academic achievement.

sedentary time and weight status [24,85,137,183-204]. One study [131] reported an effect in boys but not girls and one showed an effect in girls but not boys [139]. One study showed that among boys, being underweight was associated with more screen time [111]. The level of evidence reporting on the relationship between sedentary behaviour and body composition was of moderate quality and was classified as Level 2 with a mean Downs and Black score of 20.6 (standard deviation: \pm 1.9 ).

\section{Fitness}

Fifteen studies assessed the relationship between time spent engaging in sedentary behaviour and fitness (Table 4). Increased time spent being sedentary was associated with decreased scores for overall physical fitness, $\mathrm{VO}_{2}$ max, cardiorespiratory fitness, and musculoskeletal fitness. An intervention reported that targeting decreased sedentary behaviour lead to increases in aerobic fitness [56]. This study ( $\mathrm{n}=13$ boys and 26 girls, mean age $=$ 
Table 3 Summary table of results showing relation between sedentary behaviour and measures of body composition

\begin{tabular}{|c|c|c|c|}
\hline $\begin{array}{l}\text { Type of } \\
\text { Study }\end{array}$ & $\begin{array}{l}\text { Number of } \\
\text { Studies }\end{array}$ & $\begin{array}{l}\text { Number of } \\
\text { participants }\end{array}$ & Narrative recommendation and main findings \\
\hline $\mathrm{RCT}$ & 8 & 1886 & Reductions in sedentary behaviour are directly related to improved body composition. \\
\hline Intervention & 10 & 3547 & $\begin{array}{l}\text { TV watching and overweight/obesity were related in a dose-response manner (i.e. those who } \\
\text { watched more TV were more likely to be overweight/obese). }\end{array}$ \\
\hline Longitudinal & 33 & 85753 & $\begin{array}{l}\text { TV watching and overweight/obesity were related in a dose-response manner (i.e. those who } \\
\text { watched more TV were more likely to be overweight/obese). }\end{array}$ \\
\hline $\begin{array}{l}\text { Cross } \\
\text { sectional }\end{array}$ & 119 & 691759 & $>2$ hrs of sedentary behaviour related to increased risk of being overweight or obese. \\
\hline $\begin{array}{l}\text { Total of all } \\
\text { studies }\end{array}$ & 170 & 782884 & $\begin{array}{l}\text { Meta-analysis was performed on randomized controlled studies that looked at change in BMI. They } \\
\text { found an effect of }-0.89 \mathrm{~kg} / \mathrm{m}^{2}(95 \% \mathrm{Cl} \text { of }-1.67 \text { to }-0.11, \mathrm{p}=0.03) \text { decrease in mean BMI in the } \\
\text { intervention group. } \\
>2 \text { hrs of sedentary behaviour per day is associated with an increased risk for overweight/obesity. } \\
\text { This risk increases in a dose-response manner. } \\
\text { Each additional hour of TV viewing increased risk for obesity. }>2 \text { hrs/day significantly increased risk } \\
\text { for overweight/obesity. } \\
\text { Mean Downs and Black score }=20.9( \pm 1.9) \text {, Level } 2 \text { evidence. }\end{array}$ \\
\hline
\end{tabular}

10.5 years) showed that an intervention to decrease targeted sedentary behaviours (watching TV, playing computer games, talking on the telephone, or playing board games) led to increases in both physical activity and nontargeted sedentary behaviours. Longitudinal evidence was conflicting. One longitudinal study showed that $>2$ hours per day of TV and computer use was associated with decreased musculoskeletal fitness [205]; while the second longitudinal study found no association between increased screen time and decreased fitness. Eight of 12 cross sectional studies showed that greater than 2 hours of screen time per day was associated with decreased $\mathrm{VO}_{2}$ max, lower cardiorespiratory fitness, and lower aerobic fitness [95,206-212]. Two studies showed weak relationships between television watching and fitness $[197,213]$. Two studies showed no consistent association between television viewing and aerobic and musculoskeletal fitness $[184,214]$. The level of evidence related to fitness was classified as Level 3 with a mean Downs and Black score of 20.9 (standard deviation: \pm 2.1 ), indicating moderate quality of reporting.

Metabolic syndrome and risk for cardiovascular disease Eleven studies assessed the relationship between time spent engaging in sedentary behaviour and risk factors for MS and CVD (Table 5). All of the studies reported that increased sedentary time was associated with increased risk for MS or CVD. However, the results of these studies should be viewed with caution as the proportion of children and youth who have measurable health risk factors for MS or CVD is quite low. Longitudinal studies found that those watching more than 2 hours of television per day had higher serum cholesterol levels [88] and were more likely to have high blood pressure [215] than their peers who watched less TV. Cross sectional studies reported that high levels of screen time and self-reported sedentary behaviour were associated with increased risk for high systolic and diastolic blood pressure $[47,108,216,217]$, higher HbA1 c [218], fasting insulin [134,216], insulin resistance $[48,219]$, and MS [220]. These risk factors increase in a dose response manner with increased screen time $[216,220]$. One cross sectional study reported a significant relationship between watching TV and increased cholesterol in adolescents, but not in younger children [185]. The level of evidence for MS and CVD risk factors was classified as Level 3 with a mean Downs and Black score of 21.7 (standard deviation: \pm 2.1 ), indicating moderate quality of reporting.

Table 4 Summary table of results showing relation between sedentary behaviour and fitness

\begin{tabular}{|c|c|c|c|}
\hline $\begin{array}{l}\text { Type of } \\
\text { Study }\end{array}$ & $\begin{array}{l}\text { Number of } \\
\text { Studies }\end{array}$ & $\begin{array}{l}\text { Number of } \\
\text { participants }\end{array}$ & Narrative recommendation and main findings \\
\hline $\mathrm{RCT}$ & 0 & & \\
\hline Intervention & 1 & 76 & Reductions in sedentary behaviour lead to increased fitness. \\
\hline Longitudinal & 2 & 561 & $\begin{array}{l}\text { One study showed no association whereas one study showed higher musculoskeletal fitness in } \\
\text { those watching }<2 \text { hrs of TV per day. }\end{array}$ \\
\hline $\begin{array}{l}\text { Cross } \\
\text { sectional }\end{array}$ & 12 & 17227 & $\begin{array}{l}>2 \text { hrs of screen time per day is associated with better } \mathrm{VO}_{2} \text { max scores, better musculoskeletal } \\
\text { and cardiorespiratory fitness scores. }\end{array}$ \\
\hline $\begin{array}{l}\text { Total of all } \\
\text { studies }\end{array}$ & 15 & 17864 & $\begin{array}{l}\text { Those watching less than } 2 \text { hours of TV a day showed higher results for fitness testing and more } \\
\text { favourable bone health. } \\
\text { Mean Downs and Black score }=20.6( \pm 2.1) \text {, Level } 3 \text { evidence. }\end{array}$ \\
\hline
\end{tabular}


Table 5 Summary table of results showing relation between sedentary behaviour and markers for metabolic syndrome and cardiovascular disease

\begin{tabular}{|c|c|c|c|}
\hline $\begin{array}{l}\text { Type of } \\
\text { Study }\end{array}$ & $\begin{array}{l}\text { Number of } \\
\text { Studies }\end{array}$ & $\begin{array}{l}\text { Number of } \\
\text { participants }\end{array}$ & Narrative recommendation and main findings \\
\hline$\overline{\mathrm{RCT}}$ & 0 & & \\
\hline Longitudinal & 2 & 1675 & $\begin{array}{l}>2 \mathrm{hr} \text { of TV per day is associated with higher serum cholesterol levels. }>1.2 \mathrm{hrs} \text { of TV per day is } \\
\text { associated with increased systolic blood pressure. }\end{array}$ \\
\hline $\begin{array}{l}\text { Cross } \\
\text { sectional }\end{array}$ & 9 & 17339 & $\begin{array}{l}>2 \text { of screen time per day is associated with higher blood pressure and increased risk for } \\
\text { metabolic syndrome. }\end{array}$ \\
\hline Intervention & 0 & & \\
\hline $\begin{array}{l}\text { Total of all } \\
\text { studies }\end{array}$ & 11 & 19014 & $\begin{array}{l}\text { Increased screen time is associated with increased risk for markers of metabolic syndrome and } \\
\text { cardiovascular disease. Risk increases in a dose-response manner. } \\
\text { Mean Downs and Black score }=21.7( \pm 2.0) \text {, Level } 3 \text { evidence. }\end{array}$ \\
\hline
\end{tabular}

\section{Self esteem}

Fourteen studies assessed the relationship between time spent engaging in sedentary behaviour and self-esteem (Table 6). One RCT aimed to increase physical activity and decrease TV viewing [221], leading to a trend in improvements in self-esteem $(P=0.26)$ and concerns with body shape $(\mathrm{p}=0.03)$. Intervention studies that targeted changes in sedentary behaviour produced inverse changes in physical self-worth and self-esteem [52,54]. Cross sectional studies showed that increased screen time was associated with higher depressive symptoms, low self-esteem, and decreased perceptions of self-worth $[44,115,147,212$, 221-223]. There was evidence for a dose-response relationship as each additional hour of screen time seemed to increase the risk for lower self-esteem [147]. Two studies $[224,225]$ reported that increased TV viewing was associated with decreased self-esteem in boys but not girls, and increased aggression in girls but not boys. Two studies showed no significant relationship [226,227]. One study [228] showed a significant relationship between increased TV viewing and decreased self-esteem in adolescents but not in young children. The level of evidence for studies examining self-esteem was classified as Level 3 with a mean Downs and Black score of 21.0 (standard deviation: \pm 2.4 ) indicating moderate quality of reporting.

\section{Pro-social behaviour}

Eighteen studies assessed the relationship between time spent engaging in sedentary behaviour and pro-social behaviour (Table 7). The one longitudinal study examining the relationship between sedentary behaviour and pro-social behaviour found that sustained TV exposure (i.e. $\geq 2$ hours per day) was a significant risk factor for behavioural problems [229]. Cross sectional studies reported similar findings. Those who watched less TV were more emotionally stable, sensitive, imaginative, outgoing, self-controlled, intelligent, moralistic, college bound, and less likely to be aggressive or to engage in risky behaviour [42,115,230-235]. Two studies found a significant relationship between increased computer use and behaviour problems in boys $[111,236]$ but not girls. One study showed that increased TV viewing was associated with aggression in girls but not boys [225]. The level of evidence for studies reporting on pro-social behaviour was classified as Level 3 with a mean Downs and Black score of 19.9 (standard deviation: \pm 1.3 ) indicating moderate quality of reporting.

\section{Academic achievement}

Thirty five studies assessed the relation between time spent engaging in sedentary behaviour and academic achievement (Table 8). Academic achievement was measured in a variety of ways but included measures of I.Q., school grades, grade point average (GPA), performance on standardized tests, and self-report questionnaires (e.g. students rated their own level of academic achievement). The longitudinal studies included in this review found that children who watched higher amounts of TV had

Table 6 Summary table of results showing relation between sedentary behaviour and self-esteem

\begin{tabular}{|c|c|c|c|}
\hline $\begin{array}{l}\text { Type of } \\
\text { Study }\end{array}$ & $\begin{array}{l}\text { Number of } \\
\text { Studies }\end{array}$ & $\begin{array}{l}\text { Number of } \\
\text { participants }\end{array}$ & Narrative recommendation and main findings \\
\hline$\overline{\mathrm{RCT}}$ & 1 & 61 & $\begin{array}{l}\text { Girls who decreased sedentary behaviour had lower body dissatisfaction and showed a trend } \\
\text { towards improved self-esteem. }\end{array}$ \\
\hline Intervention & 2 & 984 & Decreases in sedentary behaviour lead to improved self worth and self-esteem. \\
\hline Longitudinal & 0 & & \\
\hline $\begin{array}{l}\text { Cross } \\
\text { sectional }\end{array}$ & 11 & 71068 & $\begin{array}{l}\text { Those with higher reported sedentary behaviour had poorer scores on self worth. This association } \\
\text { seems to increase in a dose-response manner }\end{array}$ \\
\hline $\begin{array}{l}\text { Total of all } \\
\text { studies }\end{array}$ & 14 & 72113 & $\begin{array}{l}\text { Each additional hour of TV viewing was associated with decreases in self-worth and self-concept. } \\
\text { Mean Downs and Black score }=21.0( \pm 2.4) \text {, Level } 3 \text { evidence. }\end{array}$ \\
\hline
\end{tabular}


Table 7 Summary table of results showing relation between sedentary behaviour and pro-social behaviour

\begin{tabular}{llll}
\hline $\begin{array}{l}\text { Type of } \\
\text { Study }\end{array}$ & $\begin{array}{l}\text { Number of } \\
\text { Studies }\end{array}$ & $\begin{array}{l}\text { Number of } \\
\text { participants }\end{array}$ & Narrative recommendation and main findings \\
\hline RCT & 0 & 2707 & Watching $>2$ hrs of TV per day is a risk factor for social behaviour problems \\
$\begin{array}{l}\text { Longitudinal } \\
\text { Intervention }\end{array}$ & 0 & 91934 & $\begin{array}{l}\text { Individuals watching }>3 \text { hrs of TV per day are more likely to exhibit poor social behaviours and be } \\
\text { more aggressive. Limited evidence to suggest this relationship is stronger in boys. }\end{array}$ \\
$\begin{array}{l}\text { Cross } \\
\text { sectional }\end{array}$ & 17 & 94391 & $\begin{array}{l}\text { > 2 hrs of TV per day is associated with poor pro-social behaviour. } \\
\text { Those watching less than } 3 \text { hrs of TV per day scored more positively in aspects of pro-social } \\
\text { behaviour } \\
\text { Mean Downs and Black score }=19.9( \pm 1.34) \text {, Level } 3 \text { evidence. }\end{array}$ \\
$\begin{array}{l}\text { Total of all } \\
\text { studies }\end{array}$ & 18 & &
\end{tabular}

greater difficulties with attention as teenagers [41], showed lower progression for reading level [237], and performed worse on cognitive tests [238] than those watching less than one hour of television per day. The majority of cross sectional studies (75\%) reported that children and youth who watched higher levels of TV tended to spend less time doing homework, studying, and reading for leisure which may lead to a decrease in academic achievement [42,181,239-255]. This association increased in a dose response manner [181,244,248]. Ten of the cross sectional studies found no significant relationship [57,226,227,238,256-261]. One study [228] found that this relationship was significant in adolescents but not younger children. The evidence for academic achievement was classified as Level 3 with a mean Downs and Black score of 19.2 (standard deviation: \pm 2.1 ) indicating moderate quality of reporting.

\section{Quantitative data synthesis}

Data for each of the outcomes were assessed to determine if they were sufficiently homogeneous to make meta-analysis appropriate. The only outcome for which data were consistently collected and reported and for which the characteristics of the studies were similar enough to undertake a meta-analysis was body composition. However, this was only for the RCTs; the longitudinal, cross sectional and intervention studies that examined body composition had too many inconsistencies to allow for a quantitative synthesis of results.
Change in mean BMI before and after the intervention (at the longest point of follow-up for each study) was used as the point estimate for the meta-analysis of the RCT data. Of the 8 RCTs, only 6 had data that could be used to calculate the change in BMI after the intervention $[50,58,221,262-264]$ (the other two reported on prevalence of overweight and obesity) $[57,265]$. Of the remaining six studies, one [50] examined standardized estimates of BMI only and one [262] presented only median change in BMI and not a mean change. Study authors were contacted for missing information, but no additional data was made available and thus these studies were excluded from the meta-analysis. Meta-analysis of the 4 RCTs that remained revealed an overall significant effect of $-0.89 \mathrm{~kg} / \mathrm{m}^{2}$ (95\% CI of -1.67 to -0.11 , $\mathrm{p}=0.03$ ) indicating an overall decrease in mean BMI associated with the interventions (Figure 2). The Chi square test for heterogeneity was not significant but the $\mathrm{I}^{2}$ was $46 \%$ indicating that there was low to moderate heterogeneity in the data. The funnel plot showed no indication of publication bias (data not shown).

Meta-analyses were not undertaken for other outcomes or study designs because there was substantial heterogeneity in the units of measures and type of reporting of sedentary behaviour, as well as the specific measures of each outcome. For example, when reporting on the relation between time spent watching TV and overweight and obesity, one study may report the relation between

Table 8 Summary table of results showing relation between sedentary behaviour and academic achievement

\begin{tabular}{|c|c|c|c|}
\hline $\begin{array}{l}\text { Type of } \\
\text { Study }\end{array}$ & $\begin{array}{l}\text { Number of } \\
\text { Studies }\end{array}$ & $\begin{array}{l}\text { Number of } \\
\text { participants }\end{array}$ & Narrative recommendation and main findings \\
\hline$\overline{\mathrm{RCT}}$ & 0 & & \\
\hline Longitudinal & 3 & 3530 & Watching $>1 \mathrm{hr}$ of TV per day is associated with attention difficulties. \\
\hline Intervention & 0 & & \\
\hline $\begin{array}{l}\text { Cross } \\
\text { sectional }\end{array}$ & 32 & 157637 & $>2$ hrs of screen time per day resulted in lower academic achievement. \\
\hline Intervention & 0 & & \\
\hline $\begin{array}{l}\text { Total of all } \\
\text { studies }\end{array}$ & 35 & 161167 & $\begin{array}{l}>2 \text { hrs of screen time per day is negatively associated with academic achievement. } \\
\text { Dose-response relation between time spent playing video games, watching TV and using the } \\
\text { computer (for non-academic purposes). }>3 \text { hrs/day associated with poor school performance and } \\
\text { lower I.Q. scores. } \\
\text { Mean Downs and Black score }=19.1( \pm 2.1) \text {, Level } 3 \text { evidence. }\end{array}$ \\
\hline
\end{tabular}




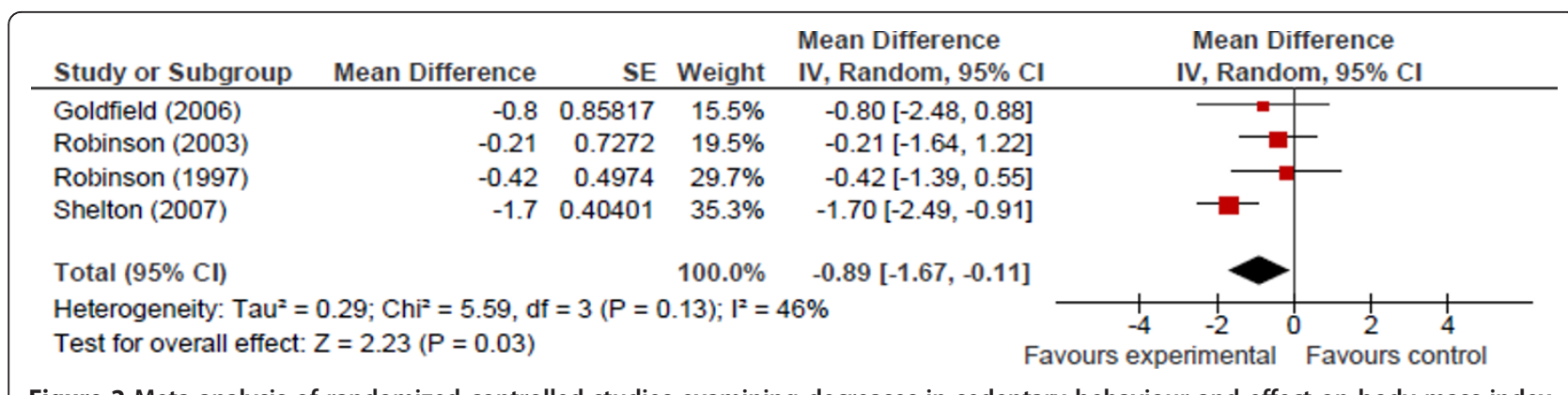

Figure 2 Meta-analysis of randomized controlled studies examining decreases in sedentary behaviour and effect on body mass index.

the frequency of TV watching and skin fold thickness, whereas another may examine the relation of daily volume of TV watching and BMI. Even for studies that examined the same outcome, for instance BMI, some would report the proportion overweight or obese, while others would report mean BMI. In addition, some studies reported on data for males or females only, while others reported only overall estimates and many were missing key information about participant characteristics or study design. As a result, we were unable to determine common point estimates and associated measures of errors for many of the studies. Due to the scope of the review, it was not feasible to contact every author for individual data to re-run the analyses. Developing reporting standards for primary studies examining the relationship between sedentary behaviour and health would help to ensure that appropriate data are available for future meta-analyses.

\section{Discussion}

Based on this systematic review of 232 studies, sedentary behaviour (assessed primarily through increased TV viewing) for more than 2 hours per day was associated with unfavourable body composition, decreased fitness, lowered scores for self-esteem and pro-social behaviour and decreased academic achievement in school-aged children and youth (5-17 years). This was true for all study designs, across all countries, using both direct and indirect measurements, and regardless of participant sample size. All studies examining risk factors for MS and CVD disease reported that increased sedentary time was associated with increased health risk; however, the included studies examined a wide range of risk factors, and thus there was insufficient evidence to draw conclusions on the relationship for metabolic risk as a whole.

High heterogeneity of the included studies limited meta-analysis to RCTs examining the relationship between television viewing and BMI. This revealed a trend to support the hypothesis that decreased time spent sedentary is associated with decreases in BMI. This result should be interpreted cautiously, given that it is only based on a small number of RCTs and that only half of the RCTs included in the review were included in the meta-analysis. Nonetheless, this meta-analysis of RCTs, which are considered to be the highest quality of research evidence, coupled with the qualitative syntheses of data from the other study designs, provides consistent evidence of the inverse relationship between sedentary behaviour and health outcomes, and that reducing sedentary behaviour can improve body composition. Furthermore, this finding was consistent with the results of observational studies and previous reviews [19-21,23,25].

Studies included in this review used primarily indirect measures (i.e. parent, teacher, and self-report questionnaires) to assess time spent engaging in sedentary behaviour. Those studies that did use direct (i.e. accelerometer) measures found that children and youth are spending a large proportion of their day (up to 9 hours) being sedentary $[24,27,29,39-47,49,178]$. Therefore, for some children and youth, a viable approach to improving health may be to work towards a reduction of at least some of their sedentary behaviours either through smaller, micro-interventions (e.g. interrupting prolonged sedentary time), or lager macro-interventions (e.g. population-based interventions and public health initiatives). Decreasing sedentary time is important for all children and youth, but it may be may be especially important to promote gradual decreases in the most sedentary group as a stepping stone to meeting sedentary behaviour guidelines [266].

\section{Strengths and limitations}

Strengths of this review included a comprehensive search strategy, a-priori inclusion and exclusion criteria and analyses, and inclusion of non-English language articles. We included direct and indirect measures of sedentary behaviour and focused on 6 diverse health indicators in children and youth. Although efforts were made to include grey literature (e.g. by contacting key informants and reviewing government documents), we did not include conference proceedings and other types of grey literature because it was impractical and unfeasible to sift through all unpublished work, and also because of limitations in 
the quality of reporting in conference abstracts $[267,268]$. We do not anticipate that additional, unpublished work would change the results.

Our study has limitations, including the types of outcome measurements and analyses reported in the primary studies and primary study quality. The scope of this review was large and included a great deal of health indicators and measurement tools. A more detailed metaanalysis would have allowed us to estimate the overall effect sizes for each outcome. However, due to the heterogeneity of the data, it was impossible to complete such analysis. Furthermore, some studies had missing information on participant characteristics making it impossible to determine if basic demographics act as a confounder for the relationship between sedentary behaviour and health. Many studies also grouped their variables into tertiles, or groups that also took into account physical activity level. Although it was still possible to ascertain information regarding the association between level of sedentary behaviour and health indicators, it made it very difficult to compare the information across studies. Similarly, very few studies measured time spent being sedentary directly (i.e. with direct observation or accelerometry). Previous work $[269,270]$ has shown significant differences between direct and indirect measures of physical activity; similar work needs to be completed with respect to sedentary behaviour to gain a better understanding of possible biases in previous studies. Indirect measurements of sedentary behaviour often lead to grouping for analyses. This may lead to bias in the results of the systematic review as many studies arbitrarily grouped their participants as "high users" if they watched more than 2 hours of television per day. This could perhaps be falsely leading us to conclude that 2 hours is the critical cut-point or threshold. Further work using direct (i.e. accelerometer) measures of sedentary behaviour and screen time as continuous variables will help to clarify if a cut-point of 2 hours is in fact biased.

The final important limitation of this review was the type of primary studies that were available for analysis. Studies with small sample sizes were excluded; however we do not believe that this had a significant impact upon the strength or direction of associations observed in this review. The majority of studies (78.4\%) included in this review were cross sectional, observational studies, using indirect (i.e. parent-, teacher, or self-report) measurements of sedentary behaviour. Cross sectional data make it impossible to infer causation and results should therefore be interpreted with caution. However, it should be noted that due to ethical considerations, it may be impossible to conduct a RCT on the effects of long periods of sedentary behaviours in children and youth. Due to the large and diverse sample sizes available in populationbased cross sectional research, and given that this information demonstrates similar trends as those seen in RCTs and intervention studies, we believe that the evidence presented in this review provides important insights into the relationship between sedentary behaviour and health outcomes in school-aged children and youth.

\section{Future work}

The purpose of this review was to provide an evidence base to inform clinical practice sedentary behaviour guidelines for children and youth [266]. Future work is needed to translate this information into clinical practice guidelines and disseminate this information to health care providers and the general public. While this review was limited to children and youth, similar work is needed to inform sedentary guidelines for young children aged 0-5 years, adults, and older adults.

As the accessibility and popularity of multiple forms of screen-based technology increases among the pediatric population, future work needs to continue to focus on media engagement. Specifically, with increasing popularity for hand-held, portable devices, 'sedentary multitasking' is becoming increasingly common. Children and youth are able to watch television, talk on the phone, and use the computer at the same time. This is a relatively new phenomenon and we are currently unaware what, if any, are the health effects associated with this high level of 'multi-screen' time. This is also true for the effect of advancements in technology and their associated health effects. For example, 'active video gaming' (e.g., Nintendo Wii ${ }^{\mathrm{TM}}$, Microsoft Kinect ${ }^{\mathrm{TM}}$, Sony's Playstation Move ${ }^{\mathrm{TM}}$ ) is advertised as an effective mode of physical activity. Although it is true that some games can require sufficient energy expenditure for health benefits [271], the socio-cognitive and physiological aspects of remaining indoors for long periods are unknown. Furthermore, children and youth can learn quite quickly how to use minimal gestures (e.g., using wrist movement only) to play the game thereby substantially reducing energy expenditure.

Finally, as described above, the vast majority of the current evidence has been based on self-report questionnaires focused on TV viewing and body composition. It is now clear that these two variables are related. Future work needs to move beyond this relationship and focus on other modes of sedentarism (e.g., prolonged sitting, passive transport) and other associated health indicators. To do this, objective measures of the time, type and context of sedentary pursuits will be needed in combination with robust and standardized measures of health indicators.

\section{Conclusions}

Physical inactivity and sedentary behaviour are pervasive and persistent public health challenges to overcome. This 
review demonstrates that there is a need to advocate for increases in physical activity AND decreases in sedentary behaviour. It is believed that a multi-level, multi-sectoral approach is required for this to be successful [11]. Ultimately, resolving the problem of inactivity requires a sustained change in individual daily activity and sedentary patterns. From a public health perspective, a reduction in sedentary behaviour may be easier than increasing physical activity per se because there are fewer restrictions (i.e. no need to change clothing or use special equipment), and can be easily attained with minimal burden to a person's time or financial resources.

This systematic review summarizes the current evidence examining the relationship between sedentary behaviours and a series of health indicators. It was determined that increased sedentary time was associated with negative health outcomes in both boys and girls; this was true across all study designs with the majority of studies $(85.8 \%)$ reporting similar relationships. The majority of current work has focused on television viewing and body composition and suggests that children and youth should watch less than 2 hours of TV per day during their discretionary time. Furthermore, children and youth should try to minimize the time they spend engaging in other sedentary pursuits throughout the day (e.g. playing video games, using the computer for non-school work or prolonged sitting). This work can be used to inform the development of evidencebased sedentary behaviour recommendations for children and youth.

\section{Additional material}

\section{Additional file 1: Search strategy.}

Additional file 2: Search strategy.

\section{List of Abbreviations}

BMl: Body Mass Index; CVD: Cardiovascular disease; DXA or DEXA: Dualenergy x-ray absorptiometry; MS: Metabolic syndrome; RCT: Randomized controlled trial; TV: Television.

\section{Acknowledgements \\ The authors are grateful to Jessie McGowan and Margaret Sampson for their contributions to this project. \\ Michelle Kho is funded by a Fellowship Award and Bisby Prize from the Canadian Institutes of Health Research. Travis Saunders is supported by a Doctoral Research Award and Richard Larouche is supported by a Banting and Best Doctoral Award from the Canadian Institutes of Health Research. Partial funding for the completion of this review came from the Public Health Agency of Canada. The views expressed herein do not necessarily represent the views of the Public Health Agency of Canada. The funders had no role in study design, data collection and analysis, decision to publish, or} preparation of the manuscript.

\section{Author details}

'Healthy Active Living and Obesity Research, Children's Hospital of Eastern Ontario Research Institute. 401 Smyth Road, Ottawa, Ontario, K1H 8L1,
Canada. ${ }^{2}$ Department of Physical Medicine and Rehabilitation, Johns Hopkins University. 600 North Wolfe Street, Baltimore, Maryland, 21202, USA. ${ }^{3}$ Office of the Task Force on Preventive Health Care, Public Health Agency of Canada. 785 Carling Avenue, Ottawa, Ontario, K1A 0K9, Canada.

\section{Authors' contributions}

MT was responsible for the initiation, conceptualization and design of the systematic review; oversaw the data collection and extraction, analysis, and interpretation of data and was responsible for revising the manuscript critically for important intellectual content. AL was responsible for conducting the search, data collection and extraction, the risk of bias assessment, analysis and interpretation of data, and drafting the manuscript. MEK was responsible for the design and methodology of the review and revising the manuscript critically for important intellectual content. SCG was responsible for the design and methodology of the manuscript, conducting the meta-analysis, and revising the manuscript critically for important intellectual content. RC, GG, TS and RL were responsible for data collection and extraction, risk of bias assessment, and were responsible for revising the manuscript critically for important intellectual content. JM was responsible for the generation of systematic review search terms. MS was responsible for methodology of the review. All authors have read and approved the final manuscript. MT is the guarantor of the paper.

\section{Competing interests}

All authors received partial financial support from the Public Health Agency of Canada; no other competing interests exist.

Received: 8 April 2011 Accepted: 21 September 2011 Published: 21 September 2011

\section{References}

1. Janssen I, LeBlanc AG: Systematic review of the health benefits of physical activity and fitness in school-aged children and youth. Int J Behav Nutr Phys Act 2010, 7(40)

2. Paterson D, Warburton D: Physical activity and functional limitations in older adults: a systematic review related to Canada's Physical Activity Guidelines. Int J Behav Nutr Phys Act 2010, 7(38).

3. Warburton D, Charlesworth S, Ivey A, Nettlefold L, Bredin S: A systematic review of the evidence for Canada's Physical Activity Guidelines for Adults. Int J Behav Nutr Phys Act 2010, 7(39)

4. U.S. Department of Health and Human Services: Physical Activity and Health: A Report of the Surgeon General. Atlanta, Georgia: U.S. Department of Health and Human Services, Centers for Disease Control and Prevention, National Center for Chronic Disease Prevention and Health Promotion; 1996.

5. World Health Organization: Global recommendations on physical activity for health. Geneva, Switzerland; World Health Organization; 2010.

6. Physical Activity Guidelines Advisory Committee: Physical Activity Guidelines Advisory Committee Report. Washington, DC: U.S. Department of Health and Human Services; 2008.

7. Colley RC, Garriguet D, Janssen I, Craig C, Clarke J, Tremblay MS: Physical activity of Canadian adults: Accelerometer results from the 2007-2009 Canadian Health Measures Survey. In Health Rep. Volume 22. Statistics Canada, Catalogue no. 82-003-XPE; 2011(1).

8. Colley RC, Garriguet D, Janssen I, Craig C, Clarke J, Tremblay MS: Physical activity of Canadian children and youth: Accelerometer results from the 2007-2009 Canadian Health Measures Survey. In Health Rep. Volume 22. Statistics Canada, Catalogue no. 82-003-XPE; 2011(1)

9. Katmarzyk PT, Tremblay MS: Limitations of Canada's physical activity data: implications for monitoring trends. Appl Physiol Nutr Metab 2007, 32: S185-S194.

10. Shields M, Tremblay MS, Laviolette M, Craig CL, Janssen I, Connor Gorber S: Fitness of Canadian adults: results from the 2007-2009 Canadian Health Measures Survey. In Health Rep. Volume 21. Statistics Canada, Catalogue no. 82-003-XPE; 2010(1).

11. Tremblay MS, Shields M, Laviolette M, Craig CL, Janssen I, Connor-Gorber S: Fitness of Canadian Children and youth: Results from the 2007-2009 Canadian Health Measures Survey. In Health Rep. Volume 21. Statistics Canada, Catalogue no. 82-003-XIE); 2010(1).

12. Troiano R, Berrigan D, Dodd K: Physical activity in the United States measured by accelerometer. Med Sci Sports Exerc 2008, 40:181-8. 
13. Flegal KM, Carroll MD, Ogden CL, Curtin LR: Prevalence and trends in obesity among US adults, 1999-2008. JAMA 2010, 303(3):235-41.

14. Walls HL, Magliano DJ, Stevenson CE, Backholder K, Mannan H, Shaw J, Peeters A: Projected progression of the prevalence of obesity in Australia. Obesity (Silver Spring), Epub 2010 Dec 13.

15. Olshansky SJ, Passaro DJ, Hershow RC, Layden J, Carnes BA, Brodt J, Hayhick L, Butler RN, Allison DB, Ludwig DS: A potential decline in life expectancy in the United States in the 21 $1^{\text {st }}$ century. N Engl J Med 2005, 352:1138-45.

16. Tremblay MS, Warburton DER, Janssen I, Patterson DH, Latimer AE, Rhodes RE, Kho ME, Hicks A, LeBlanc AG, Zehr L, Murumets K, Duggan M: New Canadian physical activity guidelines. Appl Physiol Nutr Metab 2011, 36:36-46.

17. Bull FC, Expert Working Groups: Physical activity guidelines in the U.K.: Review and recommendations. Leicestershire, UK: School of Sport, Exercise, and Health Sciences. Loughborough University; 2010.

18. Okely AD, Salmon J, Trost SG, Hinkley T: Discussion paper for the development of physical activity recommendations for children under 5 years. Australian Government Department of Health and Ageing; 2008.

19. Active Healthy Kids Canada: Healthy habits start earlier than you think Report Card on Physical Activity for Children and Youth. Toronto: Active Healthy Kids Canada; 2010.

20. Active Healthy Kids Canada: Active kids are fit to learn - Report Card on Physical Activity for Children and Youth. Toronto: Active Healthy Kids Canada; 2009.

21. Active Healthy Kids Canada: It's time to unplug our kids - Report Card on Physical Activity for Children and Youth. Toronto: Active Healthy Kids Canada; 2008.

22. Matthews CE, Chen KY, Freedson PS, Buchowski MS, Beech BM, Pate RR, Troiano R: Amount of time spent engaging in sedentary behaviours in the United States 2003-2004. Am J Epidemiol 2008, 167(7):875-81.

23. Rideout VJ, Foehr UG, Roberts DF: Generation M2: Media in the Lives of 8- to 18-Year -olds. A Kaiser Family Foundation Study. Menlo Park, California; Henry J. Kaiser Family Foundation; 2010.

24. Whitt-Glover MC, Taylor WC, Floyd MF, Yore MM, Yancey AK, Matthews CE: Disparities in physical activity and sedentary behaviors among US children and adolescents: prevalence, correlates, and intervention implications. J Public Health Policy 2009, 30(Suppl 1):S309-34.

25. Kirk SFL, Penney TL, Langille JJ: The relationship between screen time, physical activity, dietary intake and healthy weights in children and youth: literature review and recommendations for intervention. Halifax: Halifax Regional Physical Activity and the IWK Health Centre. Nova Scotia Department of Health Promotion and Protection; 2009

26. Whitlock EP, O'Connor EA, Williams SB, Beil TL, Lutz KW: Effectiveness of weight management interventions in children: A targeted systematic review for the USPSTF. Pediatrics 2010, 125:396-418.

27. Treuth MS, Baggett CD, Pratt CA, Going SB, Elder JP, Charneco EY, Webber LS: A longitudinal study of sedentary behavior and overweight in adolescent girls. Obesity (Silver Spring) 2009, 17(5):1003-8.

28. LeBlanc AG, Saunders TJ, Larouche R, Colley RC, Goldfield G, Tremblay MS: Relation between sedentary behaviours and health outcomes in children and youth. Joint Meeting of the North American Society for Pediatric Exercise Medicine and the European Group for Pediatric Work Physiology Annual General Meeting 2010, Sep 22-26, 2010; Niagara-on-the-Lake, ON. (abstract).

29. Treuth MS, Catellier DJ, Schmitz KH, Pate RR, Elder JP, McMurray RG, Blew RM, Yang S, Webber L: Weekend and weekday patterns of physical activity in overweight and normal-weight adolescent girls. Obesity (Silver Spring) 2007, 15(7):1782-8.

30. Katzmarzyk PT, Church TS, Craig CL, Bouchard C: Sitting time and mortality from all causes, cardiovascular disease, and cancer. Med Sci Sports Exerc 2009, 41:998-1005.

31. Owen N, Bauman A, Brown W: Too much sitting: a novel and important predictor of chronic disease risk? Br J Sports Med 2009, 43(2):81-3.

32. Tremblay MS, Colley R, Saunders TJ, Healy GN, Owen N: Physiological and health implications of a sedentary lifestyle. Appl Physiol Nutr Metab 2010, 35:725-40.

33. American Academy of Pediatrics: Active healthy living: Prevention of childhood obesity through increased physical activity. Pediatrics 2006, 117(5):1834-42.

34. Canadian Paediatric Society, Healthy Active Living Committee: Healthy active living for children and youth. Paediatr Child Health 2002, 7:339-45.
35. Downs SH, Black N: The feasibility of creating a checklist for the assessment of the methodological quality both of randomized and nonrandomized studies of health care interventions. J Epidemiol Community Health 1998, 52:377-84.

36. Lau DC, Douketis JD, Morrison KM, Hramiak HM, Sharma AM, Ur E: 2006 Clinical practice guidelines on the management and prevention of obesity in adults and children [summary]. CMAJ 2007, 176(8):S1-13.

37. DerSimonian R, Laird N: Meta-analysis in clinical trials. Control Clin Trials $1986,7: 177-88$

38. Higgins JPT, Thompson SG: Quantifying heterogeneity in a meta-analysis. Stat Med 2002, 21:1539-58.

39. Lampert T, Sygusch R, Schlack R, Lampert T, Sygusch R, Schlack R: [Use of electronic media in adolescence. Results of the German Health Interview and Examination Survey for Children and Adolescents (KiGGS)]. Bundesgesundheitsblatt Gesundheitsforschung Gesundheitsschutz 2007, 50(56):643-52, German.

40. Grusser SM, Thalemann R, Albrecht U, Thalemann CN: Excessive computer usage in adolescents - A psychometric evaluation. Wiener Klinische Wochenschrift 2005, 117(5-6):188-95.

41. Johnson JG, Cohen P, Kasen S, Brook JS: Extensive television viewing and the development of attention and learning difficulties during adolescence. Arch Pediatr Adolesc Med 2007, 161(5):480-6.

42. Ozmert E, Toyran M, Yurdakok K, Ozmert E, Toyran M, Yurdakok K: Behavioral correlates of television viewing in primary school children evaluated by the child behavior checklist. Arch Pediatr Adolesc Med 2002, 156(9):910-4

43. Neumann $\mathrm{H}$, Neumann P: [Nutrition and exercise of schoolchildren. Results of a cross-sectional study at the Lessing Gymnasium (secondary) school in Mannheim, Germany]. Dtsch Med Wochenschr 2007, 132(5152):2736-42, German.

44. Johnson CC, Murray DM, Elder JP, Jobe JB, Dunn AL, Kubik M, Voorhees C, Schachter K: Depressive symptoms and physical activity in adolescent girls. Med Sci Sports Exerc 2008, 40(5):818-26.

45. Nogueira JA, edo da Costa TH, Nogueira JAD, Macedo da Costa TH: Gender differences in physical activity, sedentary behavior, and their relation to body composition in active Brazilian adolescents. J Phys Act Health 2009, 6(1):93-8.

46. Poskitt EME: The evolution of childhood obesity. Int I Pediatr Obes 2010, 5(Suppl 1):11.

47. Sugiyama T, Xie D, Graham-Maar RC, Inoue K, Kobayashi Y, Stettler N: Dietary and lifestyle factors associated with blood pressure among U.S. adolescents. J Adolesc Health 2007, 40(2):166-72.

48. Sardinha LB, Andersen LB, Anderssen SA, Quiterio AL, Ornelas R, Froberg K, Riddoch $C J$, Ekelund U: Objectively measured time spent sedentary is associated with insulin resistance independent of overall and central body fat in 9- to 10-year-old Portuguese children. Diabetes Care 2008, 31(3):569-75.

49. Mitchell JA, Mattocks C, Ness AR, Leary SD, Pate RR, Dowda M, Blair SN: Sedentary behavior and obesity in a large cohort of children. Obesity (Silver Spring) 2009, 17(8):1596-602.

50. Epstein LH, Roemmich JN, Robinson JL, Paluch RA, Winiewicz DD, Fuerch $\mathrm{JH}$, Robinson TN: A randomized trial of the effects of reducing television viewing and computer use on body mass index in young children. Arch Pediatr Adolesc Med 2008, 162(3):239-45.

51. Salmon J, Ball K, Hume C, Booth M, Crawford D: Outcomes of a grouprandomized trial to prevent excess weight gain, reduce screen behaviours and promote physical activity in 10-year-old children: switchplay. Int J Obes (Lond) 2008, 32(4):601-12.

52. Goldfield GS, Mallory R, Parker T, Cunningham T, Legg C, Lumb A, Parker K, Prud'homme D, Adamo KB: Effects of modifying physical activity and sedentary behavior on psychosocial adjustment in overweight/obese children. J Pediatr Psychol 2007, 32(7):783-93.

53. Ochoa MC, Moreno-Aliaga MJ, Martinez-Gonzalez MA, Martinez JA, Marti A: Predictor factors for childhood obesity in a Spanish case-control study. Nutrition 2007, 23(5):379-84.

54. Simon C, Wagner A, DiVita C, Rauscher E, Klein-Platat C, Schweitzer B, Triby E: Intervention centred on adolescents' physical activity and sedentary behaviour (ICAPS): concept and 6-month results. Int $J$ Obes Relat Metab Disord 2004, 28(Suppl 3):S96-S103.

55. Tanasescu M, Ferris AM, Himmelgreen DA, Rodriguez N, Perez-Escamilla R: Biobehavioral factors are associated with obesity in Puerto Rican children. J Nutr 2000, 130(7):1734-42. 
56. Epstein LH, Paluch RA, Gordy CC, Dorn J: Decreasing sedentary behaviors in treating pediatric obesity. Arch Pediatr Adolesc Med 2000, 154(3):220-6

57. Gortmaker SL, Peterson K, Wiecha J, Sobol AM, Dixit S, Fox MK, Laird N: Reducing obesity via a school-based interdisciplinary intervention among youth: Planet Health. Arch Pediatr Adolesc Med 1999, 153(4):409-18

58. Robinson TN: Reducing children's television viewing to prevent obesity: a randomized controlled trial. JAMA 1999, 282(16):1561-7.

59. Epstein LH, Paluch RA, Kilanowski CK, Raynor HA: The Effect of Reinforcement or Stimulus Control to Reduce Sedentary Behavior in the Treatment of Pediatric Obesity. Health Psychol 2004, 23(4):371-80.

60. Epstein LH, Roemmich JN, Paluch RA, Raynor HA: Physical activity as a substitute for sedentary behavior in youth. Ann Behav Med 2005, 29(3):200-9.

61. Gentile DA, Welk G, Eisenmann JC, Reimer RA, Walsh DA, Russell DW, Callahan R, Walsh M, Strickland S, Fritz K: Evaluation of a multiple ecological level child obesity prevention program: Switch what you Do, View, and Chew. BMC Med 2009, 7:49.

62. Harrison M, Burns CF, McGuinness M, Heslin J, Murphy NM: Influence of a health education intervention on physical activity and screen time in primary school children: 'Switch Off-Get Active'. J Sci Med Sport 2006, 9(5):388-94.

63. Purslow LR, Hill C, Saxton J, Corder K, Wardle J: Differences in physical activity and sedentary time in relation to weight in 8-9 year old children. Int J Behav Nutr Phys Act 2008, 5(67)

64. Hesketh K, Carlin J, Wake M, Crawford D: Predictors of body mass index change in Australian primary school children. Int J Pediatr Obes 2009, 4(1):45-53

65. Timperio A, Salmon J, Ball K, Baur LA, Telford A, Jackson M, Salmon L, Crawford D: Family physical activity and sedentary environments and weight change in children. Int J Pediatr Obes 2008, 3(3):160-7.

66. Danner FW: A national longitudinal study of the association between hours of TV viewing and the trajectory of BMI growth among US children. J Pediatr Psychol 2008, 33(10):1100-7.

67. Henderson VR: Longitudinal associations between television viewing and body mass index among white and black girls. J Adolesc Health 2007, 41(6):544-50

68. Blair NJ, Thompson JM, Black PN, Becroft DM, Clark PM, Han DY, Robinson E, Waldie KE, Wild CJ, Mitchell EA: Risk factors for obesity in 7year-old European children: the Auckland Birthweight Collaborative Study. Arch Dis Child 2007, 92(10):866-71.

69. O'Brien M, Nader PR, Houts RM, Bradley R, Friedman SL, Belsky J, Susman E: The ecology of childhood overweight: a 12-year longitudinal analysis. Int J Obes (Lond) 2007, 31(9):1469-78.

70. Gable S, Chang Y, Krull JL: Television watching and frequency of family meals are predictive of overweight onset and persistence in a national sample of school-aged children. J Am Diet Assoc 2007, 107(1):53-61.

71. Burke V, Beilin LJ, Durkin K, Stritzke WG, Houghton S, Cameron CA: Television, computer use, physical activity, diet and fatness in Australian adolescents. Int J Pediatr Obes 2006, 1(4):248-55.

72. Hancox RJ, Poulton R: Watching television is associated with childhood obesity: but is it clinically important? Int J Obes (Lond) 2006, 30(1):171-5.

73. Janz KF, Burns TL, Levy SM, lowa Bone DS: Tracking of activity and sedentary behaviors in childhood: the lowa Bone Development Study. Am J Prev Med 2005, 29(3):171-8.

74. Parsons TJ, Power C, Manor O: Physical activity, television viewing and body mass index: a cross-sectional analysis from childhood to adulthood in the 1958 British cohort. Int J Obes (Lond) 2005, 29(10):1212-21.

75. Kaur H, Choi WS, Mayo MS, Harris KJ: Duration of television watching is associated with increased body mass index. J Pediatr 2003, 143(4):506-11.

76. Berkey CS, Rockett HR, Gillman MW, Colditz GA: One-year changes in activity and in inactivity among 10- to 15 -year-old boys and girls: relationship to change in body mass index. Pediatrics 2003, 111(4):836-43.

77. Bhargava A, Jolliffe D, Howard LL: Socio-economic, behavioural and environmental factors predicted body weights and household food insecurity scores in the Early Childhood Longitudinal StudyKindergarten. Br J Nutr 2008, 100(2):438-44.

78. Chen JL, Wall D, Kennedy C, Unnithan V, Yeh CH: Predictors of increased body mass index in Chinese children. Prog Cardiovasc Nurs 2007, 22(3):138-44.
79. Elgar FJ, Roberts C, Moore L, Tudor-Smith C: Sedentary behaviour, physical activity and weight problems in adolescents in Wales. Public Health 2005, 119(6):518-24

80. Hesketh $\mathrm{K}$, Wake M, Graham M, Waters E: Stability of television viewing and electronic game/computer use in a prospective cohort study of Australian children: Relationship with body mass index. Int I Behav Nutr Phys Act 2007, 4(60)

81. Lajunen HR, Keski-Rahkonen A, Pulkkinen L, Rose RJ, Rissanen A, Kaprio J: Leisure activity patterns and their associations with overweight: a prospective study among adolescents. J Adolesc 2009, 32(5):1089-103.

82. Jago R, Baranowski T, Baranowski JC, Thompson D, Greaves KA: BMI from 3-6 $\mathrm{y}$ of age is predicted by TV viewing and physical activity, not diet. Int J Obes (Lond) 2005, 29(6):557-64.

83. Aires L, Andersen LB, Mendonca D, Martins C, Silva G, Mota J: A 3-year longitudinal analysis of changes in fitness, physical activity, fatness and screen time. Acta Paediatr 2010, 99(1):140-4, Erratum in: Acta Paediatr. 2010, 99 (1):153.

84. Fulton JE, Dai S, Steffen LM, Grunbaum JA, Shah SM, Labarthe DR: Physical activity, energy intake, sedentary behavior, and adiposity in youth. Am J Prev Med 2009, 37(Suppl 1):S40-9.

85. Day RS, Fulton JE, Dai S, Mihalopoulos NL, Barradas DT: Nutrient intake, physical activity, and CVD risk factors in children: Project HeartBeat! Am J Prev Med 2009, 37(1 Suppl):S25-S33.

86. Borradaile KE, Foster GD, May H, Karpyn A, Sherman S, Grundy K, Nachmani J, Vander Veur S, Boruch RF: Associations between the Youth/ Adolescent Questionnaire, the Youth/Adolescent Activity Questionnaire, and body mass index $\mathrm{z}$ score in low-income inner-city fourth through sixth grade children. Am J Clin Nutr 2008, 87(6):1650-5.

87. Must A, Bandini LG, Tybor DJ, Phillips SM, Naumova EN, Dietz WH: Activity, inactivity, and screen time in relation to weight and fatness over adolescence in girls. Obesity (Silver Spring) 2007, 15(7):1774-81.

88. Hancox RJ, Milne BJ, Poulton R: Association between child and adolescent television viewing and adult health: a longitudinal birth cohort study. Lancet 2004, 364(9430):257-62.

89. Maffeis C, Talamini G, Tato L: Influence of diet, physical activity and parents' obesity on children's adiposity: a four-year longitudinal study. Int J Obes Relat Metab Disord 1998, 22(8):758-64.

90. Berkey CS, Rockett HR, Field AE, Gillman MW, Frazier AL, Camargu CA Jr, Colditz GA: Activity, dietary intake, and weight changes in a longitudinal study of preadolescent and adolescent boys and girls. Pediatrics 2000, 105(4):E56.

91. Sun Y, Sekine M, Kagamimori S: Lifestyle and overweight among Japanese adolescents: the Toyama Birth Cohort Study. J Epidemiol 2009, 19(6):303-10

92. Lajous M, Chavarro J, Peterson KE, Hernandez-Prado B, Cruz-Valdez A Hernandez-Avila M, Lazcano-Ponce E: Screen time and adiposity in adolescents in Mexico. Public Health Nutr 2009, 12(10):1938-45.

93. Nagel G, Wabitsch M, Galm C, Berg S, Brandstetter S, Fritz M, Klenk J, Peter R, Prokopchuk D, Steiner R, Stroth S, Wartha O, Weiland SK, Steinacker J: Determinants of obesity in the Ulm Research on Metabolism, Exercise and Lifestyle in Children (URMEL-ICE). Eur J Pediatr 2009, 168(10):1259-67.

94. Morgenstern M, Sargent JD, Hanewinkel R: Relation between socioeconomic status and body mass index: evidence of an indirect path via television use. Arch Pediatr Adolesc Med 2009, 163(8):731-8.

95. Christoforidis A, Papadopoulou E, Dimitriadou M, Stilpnopoulou D, Gkogka C, Katzus G, Thanassiou-Metaxa M: Reference values for quantitative ultrasonography (QUS) of radius and tibia in healthy Greek pediatric population: clinical correlations. J Clin Densitom 2009, 12(3):360-8.

96. Bukara-Radujkovic G, Zdravkovic D, Bukara-Radujkovic G, Zdravkovic D: Physical activity as an important determinant in developing childhood obesity. Med Pregl 2009, 62(3-4):107-13.

97. Vader AM, Walters ST, Harris TR, Hoelscher DM: Television viewing and snacking behaviors of fourth- and eighth-grade schoolchildren in Texas. Prev Chronic Dis 2009, 6(3):A89.

98. Steffen LM, Dai S, Fulton JE, Labarthe DR: Overweight in children and adolescents associated with TV viewing and parental weight: Project HeartBeat! Am J Prev Med 2009, 37(Suppl 1):S50-5.

99. Padez C, Mourao I, Moreira P, Rosado V: Long sleep duration and childhood overweight/obesity and body fat. Am J Human Biol 2009, 21(3):371-6. 
100. Wong SL, Leatherdale ST: Association between sedentary behavior, physical activity, and obesity: inactivity among active kids. Prev Chronic Dis 2009, 6(1):A26.

101. Pratt C, Webber LS, Baggett CD, Ward D, Pate RR, Loham T, Lytle L, Elder JP. Sedentary activity and body composition of middle school girls: the trial of activity for adolescent girls. Res Q Exerc Sport 2008, 79(4):458-67.

102. Tovar G, Poveda JG, Pinilla MI, Lobelo F: [Relationship between overweight, physical activity and physical fitness in school-aged boys in Bogota Colombia]. Arch Latinoam Nutr 2008, 58(3):265-73, Spanish.

103. Anderson SE, Economos CD, Must A: Active play and screen time in US children aged 4 to 11 years in relation to sociodemographic and weight status characteristics: a nationally representative cross-sectional analysis. BMC Public Health 2008, 8:366.

104. Boone-Heinonen J, Gordon-Larsen P, Adair LS: Obesogenic clusters: multidimensional adolescent obesity-related behaviors in the U.S. Ann Behav Med 2008, 36(3):217-30.

105. Beyerlein A, Fahrmeir L, Mansmann U, Toschke AM: Alternative regression models to assess increase in childhood BMI. BMC Med Res Methodol 2008, 8:59.

106. Singh GK, Kogan MD, Van Dyck PC, Siahpush M: Racial/ethnic, socioeconomic, and behavioral determinants of childhood and adolescent obesity in the United States: analyzing independent and joint associations. Ann Epidemiol 2008, 18(9):682-95.

107. Laurson KR, Eisenmann JC, Welk GJ, Wickel EE, Gentile DA, Walsh DA: Combined influence of physical activity and screen time recommendations on childhood overweight. J Pediatr 2008, 153(2):209-14.

108. Wells JC, Hallal PC, Reichert FF, Menezes AM, Araujo CL, Victora CG: Sleep patterns and television viewing in relation to obesity and blood pressure: evidence from an adolescent Brazilian birth cohort. Int J Obes (Lond) 2008, 32(7):1042-9.

109. Basaldua N, Chiquete E, Basaldua N, Chiquete E: Common predictors of excessive adiposity in children from a region with high prevalence of overweight. Ann Nutr Metab 2008, 52(3):227-32.

110. Vicente-Rodriguez G, Rey-Lopez JP, Martin-Matillas M, Moreno LA, Warnberg J, Redondo C, Tercedor P, Delgado M, Marcos A, Castillo M, Bueno M, AVENA Study Group: Television watching, videogames, and excess of body fat in Spanish adolescents: The AVENA study. Nutrition 2008, 24(7-8):654-62

111. Leatherdale ST, Wong SL: Modifiable characteristics associated with sedentary behaviours among youth. Int J Pediatr Obes 2008, 3(2):93-101.

112. Toschke AM, Von KR, Beyerlein A, Ruckinger S: Risk factors for childhood obesity: shift of the entire BMI distribution vs. shift of the upper tail only in a cross sectional study. BMC Public Health 2008, 8:115.

113. Eisenmann JC, Bartee RT, Smith DT, Welk GJ, Fu Q: Combined influence of physical activity and television viewing on the risk of overweight in US youth. Int J Obes (Lond) 2008, 32(4):613-8.

114. Maffeis C, Grezzani A, Perrone L, Del Giudice EM, Saggese G, Tato L: Could the savory taste of snacks be a further risk factor for overweight in children? J Pediatr Gastroenterol Nutr 2008, 46(4):429-37.

115. Chen MY, Liou YM, Wu JY: The relationship between TV/computer time and adolescents' health-promoting behavior: a secondary data analysis. J Nurs Res 2008, 16(1):75-85.

116. Lasserre AM, Chiolero A, Cachat F, Paccaud F, Bovet P: Overweight in Swiss children and associations with children's and parents' characteristics. Obesity (Silver Spring) 2007, 15(12):2912-9.

117. Kuriyan R, Bhat $S$, Thomas T, Vaz M, Kurpad AV: Television viewing and sleep are associated with overweight among urban and semi-urban South Indian children. Nutr J 2007, 6:25.

118. Lutfiyya MN, Lipsky MS, Wisdom-Behounek J, Inpanbutr-Martinkus M: Is rural residency a risk factor for overweight and obesity for U.S. children? Obesity (Silver Spring) 2007, 15(9):2348-56.

119. Butte NF, Puyau MR, Adolph AL, Vohra FA, Zakeri I: Physical activity in nonoverweight and overweight Hispanic children and adolescents. Med Sci Sports Exerc 2007, 39(8):1257-66.

120. Wang Y, Liang H, Tussing L, Braunschweig C, Caballero B, Flay B: Obesity and related risk factors among low socio-economic status minority students in Chicago. Public Health Nutr 2007, 10(9):927-38.

121. Toschke AM, Ruckinger S, Bohler E, Von KR: Adjusted population attributable fractions and preventable potential of risk factors for childhood obesity. Public Health Nutr 2007, 10(9):902-6.
122. Ortega FB, Tresaco B, Ruiz JR, Moreno LA, Martin-Matillas M, Mesa JL, Warnberg J, Bueno M, Tercedor P, Gutierrez A, Castillo MJ, AVENA Study Group: Cardiorespiratory fitness and sedentary activities are associated with adiposity in adolescents. Obesity (Silver Spring) 2007, 15(6):1589-99.

123. Bellisle F, Rolland-Cachera MF, Kellogg Scientific Advisory Committee: Three consecutive $(1993,1995,1997)$ surveys of food intake, nutritional attitudes and knowledge, and lifestyle in 1000 French children, aged 911 years. J Hum Nutr Diet 2007, 20(3):241-51.

124. Spinks AB, Macpherson AK, Bain C, McClure RJ: Compliance with the Australian national physical activity guidelines for children: relationship to overweight status. J Sci Med Sport 2007, 10(3):156-63.

125. Delva J, O'Malley PM, Johnston LD: Health-related behaviors and overweight: a study of Latino adolescents in the United States of America. Rev Panam Salud Publica 2007, 21(1):11-20.

126. Ramos E, Barros H: Family and school determinants of overweight in 13year-old Portuguese adolescents. Acta Paediatr 2007, 96(2):281-6.

127. Lioret S, Maire B, Volatier JL, Charles MA: Child overweight in France and its relationship with physical activity, sedentary behaviour and socioeconomic status. Eur J Clin Nutr 2007, 61(4):509-16.

128. Lajunen HR, Keski-Rahkonen A, Pulkkinen L, Rose RJ, Rissanen A, Kaprio J: Are computer and cell phone use associated with body mass index and overweight? A population study among twin adolescents. BMC Public Health 2007, 7:24.

129. te Velde SJ, De B, Thorsdottir I, Rasmussen M, Hagstromer M, Klepp KI, Brug J: Patterns in sedentary and exercise behaviors and associations with overweight in 9-14-year-old boys and girls-a cross-sectional study. BMC Public Health 2007, 7:16.

130. Boutelle KN, Hannan PJ, Neumark-Sztainer D, Himes JH: Identification and correlates of weight loss in adolescents in a national sample. Obesity (Silver Spring) 2007, 15(2):473-82.

131. Carvalhal MM, Padez MC, Moreira PA, Rosado VM: Overweight and obesity related to activities in Portuguese children, 7-9 years. Eur J Public Health 2007, 17(1):42-6.

132. Zabinski MF, Norman GJ, Sallis JF, Calfas KJ, Patrick K: Patterns of sedentary behavior among adolescents. Health Psychol 2007, 26(1):113-20.

133. Hardy LL, Dobbins T, Booth ML, ney-Wilson E, Okely AD: Sedentary behaviours among Australian adolescents. Aust N Z J Public Health 2006, 30(6):534-40.

134. Ekelund U, Brage S, Froberg K, Harro M, Anderssen SA, Sardinha LB, Riddoch C, Andersen LB: TV viewing and physical activity are independently associated with metabolic risk in children: the European Youth Heart Study. PLoS Med 2006, 3(12):e488.

135. Moraes SA, Beltran RJ, Mondini L, Freitas IC: [Prevalence of overweight and obesity, and associated factors in school children from urban area in Chilpancingo, Guerrero, Mexico, 2004]. Cad Saude Publica 2006, 22(6):1289-301, Portuguese.

136. Salmon J, Campbell KJ, Crawford DA: Television viewing habits associated with obesity risk factors: a survey of Melbourne schoolchildren. Med $J$ Aust 2006, 184(2):64-7.

137. Violante R, del Rio Navarro BE, Berber A, Ramirez CN, Baeza BM, Sienra Monge JJ: Obesity risk factors in the ISAAC (International Study of Asthma and Allergies in Childhood) in Mexico City. Rev Alerg Mex 2005, 52(4):141-5.

138. Rapp K, Schick KH, Bode H, Weiland SK: Type of kindergarten and other potential determinants of overweight in pre-school children. Public Health Nutr 2005, 8(6):642-9.

139. Kautiainen S, Koivusilta L, Lintonen T, Virtanen SM, Rimpela A: Use of information and communication technology and prevalence of overweight and obesity among adolescents. Int J Obes (Lond) 2005, 29(8):925-33.

140. Giammattei J, Blix G, Marshak HH, Wollitzer AO, Pettitt DJ: Television watching and soft drink consumption: associations with obesity in 11to 13-year-old schoolchildren. Arch Pediatr Adolesc Med 2003, 157(9):882-6.

141. Ruangdaraganon N, Kotchabhakdi N, Udomsubpayakul U, Kunanusont C, Suriyawongpaisal $P$ : The association between television viewing and childhood obesity: a national survey in Thailand. J Med Assoc Thai 2002, 85(Suppl 4):S1075-80.

142. Andersen RE, Crespo CJ, Bartlett SJ, Cheskin LJ, Pratt M: Relationship of physical activity and television watching with body weight and level of fatness among children: results from the Third National Health and Nutrition Examination Survey. JAMA 1998, 279(12):938-42. 
143. Gortmaker SL, Must A, Sobol AM, Peterson K, Colditz GA, Dietx WH: Television viewing as a cause of increasing obesity among children in the United States, 1986-1990. Arch Pediatr Adolesc Med 1996, 150(4):356-62.

144. Hirschler V, Buzzano K, Erviti A, Ismael N, Silva S, Dalamon R: Overweight and lifestyle behaviors of low socioeconomic elementary school children in Buenos Aires. BMC Pediatrics 2009, 9(1).

145. Tsai H-J, Tsai AC: The association of BMI and sedentary time with respiratory symptoms and asthma in 5th grade schoolchildren in Kaohsiung, Taiwan. J Asthma 2009, 46(1):9-15.

146. Trang NHHD, Hong TK, Dibley MJ, Sibbritt DW: Factors associated with physical inactivity in adolescents in Ho Chi Minh City, Vietnam. Med SCi Sports Exerc 2009, 41(7):1374-83.

147. Russ SA, Larson K, Franke TM, Halfon N: Associations between media use and health in US children. Acad Pediatr 2009, 9(5):300-6.

148. Nawal LM, Garcia R, Dankwa CM, Young T, Lipsky MS: Overweight and obese prevalence rates in African American and Hispanic children: An analysis of data from the 2003-2004 National Survey of Children's Health. J Am Board Fam Med 2008, 21(3):191-9.

149. Collins $A E$, Pakiz B, Rock CL: Factors associated with obesity in Indonesian adolescents. Int J Pediatr Obes 2008, 3(1):58-64.

150. Gomez LF, Parra DC, Lobelo F, Samper B, Moreno J, Jacoby E, Lucumi DI, Matsudo S, Borda C: Television viewing and its association with overweight in Colombian children: Results from the 2005 National Nutrition Survey: A cross sectional study. Int J Behav Nutr Phys Act 2007, 4(41).

151. Barlow SE, Chang J-J: Is parental aggravation associated with childhood overweight? An analysis of the national survey of children's health 2003. Acta Paediatrica 2007, 96(9):1360-2.

152. Utter J, Scragg R, Schaaf D, Fitzgerald E, Wilson N: Correlates of body mass index among a nationally representative sample of New Zealand children. Int J Pediatr Obes 2007, 2(2):104-13.

153. Tsai H-J, Tsai AC, Nriagu J, Ghosh D, Gong M, Sandretto A: Associations of BMI, TV-watching time, and physical activity on respiratory symptoms and asthma in 5th grade schoolchildren in Taipei, Taiwan. J Asthma 2007, 44(5):397-401.

154. Chaput J-P, Brunet M, Tremblay A: Relationship between short sleeping hours and childhood overweight/obesity: Results from the 'Quebec en Forme' project. Int J Obes (Lond) 2006, 30(7):1080-5.

155. Andersen LF, Lillegaard ITL, Overby N, Lytle L, Klepp K-I, Johansson L: Overweight and obesity among Norwegian schoolchildren: Changes from 1993 to 2000. Scand J Public Health 2005, 33(2):99-106.

156. Gibson S, Lambert J, Neate D: Associations between weight status, physical activity, and consumption of biscuits, cakes and confectionery among young people in Britain. Nutrition Bulletin 2004, 29(4):301-9.

157. Da CR, Taddei JAAC, Colugnatti F: Obesity among children attending elementary public schools in Sao Paulo, Brazil: A case-control study. Public Health Nutr 2003, 6(7):659-63.

158. Taylor WC, Sallis JF, Dowda M, Freedson PS, Eason K, Pate RR: Activity patterns and correlates among youth: Differences by weight status. Pediatr Exerc Sci 2002, 14(4):418-31.

159. van Zutphen $M$, Bell $A C$, Kremer PJ, Swinburn BA: Association between the family environment and television viewing in Australian children. $J$ Paediatr Child Health 2007, 43(6):458-63.

160. Lagiou A, Parava M: Correlates of childhood obesity in Athens, Greece. Public Health Nutr 2008, 11(9):940-5.

161. Smith BJ, Phongsavan P, Havea D, Halavatau V, Chey T: Body mass index, physical activity and dietary behaviours among adolescents in the Kingdom of Tonga. Public Health Nutr 2007, 10(2):137-44.

162. Shields M: Overweight and obesity among children and youth. Health Rep 2006, 17(3):27-42, Statistics Canada, Catalogue no. 82-003.

163. Aucote HM, Cooper A: Relationships between body fatness, small-screen sedentary activity and regionality among schoolchildren in Victoria, Australia. Aust J Rural Health 2009, 17(3):141-6.

164. Boone JE, Gordon-Larsen P, Adair LS, Popkin BM: Screen time and physical activity during adolescence: Longitudinal effects on obesity in young adulthood. Int J Behav Nutr Phys Act 2007, 4(26)

165. Klein-Platat C, Oujaa M, Wagner A, Haan MC, Arveiler D, Schlienger JL, Simon C: Physical activity is inversely related to waist circumference in 12-y-old French adolescents. Int J Obes (Lond) 2005, 29(1):9-14.
166. Janssen I, Katzmarzyk PT, Boyce WF, King MA, Pickett W: Overweight and obesity in Canadian adolescents and their associations with dietary habits and physical activity patterns. J Adolesc Health 2004, 35(5):360-7.

167. Graf C, Koch B, Dordel S, Schindler-Marlow S, Icks A, Schuller A, BjarnasonWehrens B, Tokarski W, Predel HG: Physical activity, leisure habits and obesity in first-grade children. Eur J Cardiovasc Prev Rehabil 2004, 11(4):284-90.

168. Stettler N, Signer TM, Suter PM: Electronic games and environmental factors associated with childhood obesity in Switzerland. Obes Res 2004, 12(6):896-903.

169. Patrick K, Norman GJ, Calfas KJ, Sallis JF, Zabinski MF: Diet, physical activity, and sedentary behaviors as risk factors for overweight in adolescence. Arch Pediatr Adolesc Med 2004, 158(4):385-90.

170. Vandewater EA, Shim MS, Caplovitz AG: Linking obesity and activity level with children's television and video game use. J Adolesc 2004, 27(1):71-85.

171. Utter J, Neumark-Sztainer D, Jeffery R, Story M: Couch potatoes or french fries: are sedentary behaviors associated with body mass index, physical activity, and dietary behaviors among adolescents? J Am Diet Assoc 2003, 103(10):1298-305.

172. Tremblay MS, Willms JD: Is the Canadian childhood obesity epidemic related to physical inactivity? Int J Obes Relat Metab Disord 2003, 27(9):1100-5.

173. Lowry R, Wechsler H, Galuska DA, Fulton JE, Kann L: Television viewing and its associations with overweight, sedentary lifestyle, and insufficient consumption of fruits and vegetables among US high school students: differences by race, ethnicity, and gender. J Sch Health 2002, 72(10):413-21.

174. Janz KF, Levy SM, Burns TL, Torner JC, Willing MC, Warren JJ: Fatness, physical activity, and television viewing in children during the adiposity rebound period: the lowa Bone Development Study. Prev Med 2002, 35(6):563-71.

175. Eisenmann JC, Bartee RT, Wang MQ: Physical activity, TV viewing, and weight in U.S. youth: 1999 Youth Risk Behavior Survey. Obes Res 2002, 10(5):379-85.

176. Gordon-Larsen P, Adair LS, Popkin BM: Ethnic differences in physical activity and inactivity patterns and overweight status. Obes Res 2002, 10(3):141-9.

177. Crespo CJ, Smit E, Troiano RP, Bartlett SJ, Macera CA, Anderson RE: Television watching, energy intake, and obesity in US children: results from the third National Health and Nutrition Examination Survey, 19881994. Arch Pediatr Adolesc Med 2001, 155(3):360-5.

178. Hernandez B, Gortmaker SL, Colditz GA, Peterson KE, Laird NM, ParraCabrera S: Association of obesity with physical activity, television programs and other forms of video viewing among children in Mexico city. Int J Obes Relat Metab Disord 1999, 23(8):845-54.

179. Muller MJ, Koertzinger I, Mast M, Langnase K, Grund A: Physical activity and diet in 5 to 7 years old children. Public Health Nutr 1999, 2(3A):443-4, Erratum in: Public Health Nutr. 2000;3(1):443-4.

180. Obarzanek E, Schreiber GB, Crawford PB, Goldman SR, Barrier PM, Frederick MM, Lakatos E: Energy intake and physical activity in relation to indexes of body fat: the National Heart, Lung, and Blood Institute Growth and Health Study. Am J Clin Nutr 1994, 60(1):15-22.

181. Dietz WH Jr, Gortmaker SL: Do we fatten our children at the television set? Obesity and television viewing in children and adolescents. Pediatrics 1985, 75(5):807-12.

182. da Silva RC, Malina RM: [Level of physical activity in adolescents from Niteroi, Rio de Janeiro, Brazil]. Cad Saude Publica 2000, 16(4):1091-7, Portuguese.

183. Asante PA, Cox J, Sonneville K, Samuels RC, Taveras EM: Overweight prevention in pediatric primary care: a needs assessment of an urban racial/ethnic minority population. Clin Pediatr (Phila) 2009, 48(8):837-43.

184. Katzmarzyk PT, Malina RM, Song TM, Bouchard C: Television viewing, physical activity, and health-related fitness of youth in the Quebec Family Study. J Adolesc Health 1998, 23(5):318-25.

185. Purath J, Lansinger T, Ragheb C: Cardiac risk evaluation for elementary school children. Public Health Nurs 1995, 12(3):189-95.

186. Wake M, Hesketh K, Waters E: Television, computer use and body mass index in Australian primary school children. J Paediatr Child Health 2003, 39(2):130-4. 
187. McMurray RG, Harrell JS, Deng S, Bradley CB, Cox LM, Bandiwala SI: The influence of physical activity, socioeconomic status, and ethnicity on the weight status of adolescents. Obes Res 2000, 8(2):130-9.

188. Forshee RA, Anderson PA, Storey ML: Associations of various family characteristics and time use with children's body mass index. $J$ Community Health Nurs 2009, 26(2):77-86.

189. Thompson AM, Campagna PD, Durant M, Murphy RJ, Rehman LA, Wadsworth SA: Are overweight students in Grades 3, 7, and 11 less physically active than their healthy weight counterparts? Int J Pediatr Obes 2009, 4(1):28-35.

190. Hume C, Singh A, Brug J, Mechelen W, Chinapaw M: Dose-response associations between screen time and overweight among youth. Int J Pediatr Obes 2009, 4(1):61-4.

191. Alves JG, Siqueira PP, Figueiroa JN: Overweight and physical inactivity in children living in favelas in the metropolitan region of Recife, Brazil. J Pediatr (Rio J) 2009, 85(1):67-71.

192. Al SH, Vereecken C, Abdeen Z, Coats E, Maes L: Associations of overweight and of weight dissatisfaction among Palestinian adolescents: findings from the national study of Palestinian schoolchildren. J Hum Nutr Diet 2009, 22(1):40-9

193. Mihas C, Mariolis A, Manios Y, Naska A, Panagiotakos D, Arapaki A, Alevizos A, Mariolis-Sapsakos T, Tountas Y: Overweight/obesity and factors associated with body mass index during adolescence: the VYRONAS study. Acta Paediatr 2009, 98(3):495-500.

194. Mikolajczyk RT, Richter M: Associations of behavioural, psychosocial and socioeconomic factors with over- and underweight among German adolescents. Int J Public Health 2008, 53(4):214-20.

195. Islam-Zwart K, Cawston A: Investigation of factors contributing to diabetes risk in American Indian/Alaska native youth. Am Indian Alsk Native Ment Health Res 2008, 14(3):49-58.

196. Kosti Rl, Panagiotakos DB, Mihas CC, Alevizos A, Zampelas A, Mariolis A, Tountas Y: Dietary habits, physical activity and prevalence of overweight/obesity among adolescents in Greece: the Vyronas study Med Sci Monit 2007, 13(10):CR437-CR444.

197. Katzmarzyk PT, Malina RM, Song TM, Bouchard C: Physical activity and health-related fitness in youth: a multivariate analysis. Med Sci Sports Exerc 1998, 30(5):709-14.

198. Vaughan C, Kilkkinen A, Heistaro S, Laatikainen T, Dunbar J: The dietary, physical activity and sedentary behaviours of Victorian regional secondary school students. Aust J Prim Health 2007, 13(1):81-8.

199. Mota J, Ribeiro J, Santos MP, Gomes H: Obesity, physical activity, computer use, and TV viewing in Portuguese adolescents. Pediatr Exerc Sci 2006, 18(1):113-21.

200. Colwell J, Kato M: Investigation of the relationship between social isolation, self-esteem, aggression and computer game play in Japanese adolescents. Asian Journal of Social Psychology 2003, 6(2):Aug-158.

201. Forshee RA, Anderson PA, Storey ML: The role of beverage consumption, physical activity, sedentary behavior, and demographics on body mass index of adolescents. Int J Food Sci Nutr 2004, 55(6):463-78.

202. Waller CE, Du S, Popkin BM: Patterns of overweight, inactivity, and snacking in Chinese children. Obes Res 2003, 11(8):957-61.

203. Wolf AM, Gortmaker SL, Cheung L, Gray HM, Herzog DB, Colditz GA: Activity, inactivity, and obesity: racial, ethnic, and age differences among schoolgirls. Am J Public Health 1993, 83(11):1625-7.

204. Robinson TN, Hammer LD, Killen JD, Kraemer HC, Wilson DM, Hayward C, Taylor CB: Does television viewing increase obesity and reduce physical activity? Cross-sectional and longitudinal analyses among adolescent girls. Pediatrics 1993, 91(2):273-80.

205. Wosje KS, Khoury PR, Claytor RP, Copeland KA, Kalkwarf HJ, Daniels SR: Adiposity and TV viewing are related to less bone accrual in young children. J Pediatr 2009, 154(1):79-85.

206. Tucker $L A$ : The relationship of television viewing to physical fitness and obesity. Adolescence 1986, 21(84):797-806.

207. Albarwani S, Al-Hashmi K, Al-Abri M, Jaju D, Hassan MO: Effects of overweight and leisure-time activities on aerobic fitness in urban and rural adolescents. Metab 2009, 7(4):369-74.

208. Lobelo F, Dowda M, Pfeiffer KA, Pate RR: Electronic media exposure and its association with activity-related outcomes in female adolescents: cross-sectional and longitudinal analyses. J Phys Act Health 2009, 6(2):137-43.
209. Hardy LL, Dobbins TA, ney-Wilson EA, Okely AD, Booth ML: Sedentariness, small-screen recreation, and fitness in youth. Am J Prev Med 2009, 36(2):120-5.

210. Pate RR, Wang CY, Dowda M, Farrell SW, O'Neill JR: Cardiorespiratory fitness levels among US youth 12 to 19 years of age: findings from the 1999-2002 National Health and Nutrition Examination Survey. Arch Pediatr Adolesc Med 2006, 160(10):1005-12.

211. Dollman J, Ridley K: Differences in body fatness, fat patterning and cardio-respiratory fitness between groups of Australian children formed on the basis of physical activity and television viewing guidelines. J Phys Act Health 2006, 3(2):191-199.

212. Tucker LA: Television, teenagers, and health. J Youth Adolesc 1987, 16(5):415-25.

213. Armstrong CA, Sallis JF, Alcaraz JE, Kolody B, McKenzie TL, Hovell MF: Children's television viewing, body fat, and physical fitness. Am J Health Promot 1998, 12(6):363-8.

214. Tucker LA, Hager RL, Tucker LA, Hager RL: Television viewing and muscular fitness of children. Percept Mot Skills 1996, 82(3 Pt 2):1316-8.

215. Dasgupta K, O'Loughlin J, Chen S, Karp I, Paradis G, Tremblay J, Hamet P, Pilote L: Emergence of sex differences in prevalence of high systolic blood pressure: analysis of a longitudinal adolescent cohort. Circulation 2006, 114(24):2663-70, Erratum in: Circulation 2007, 116 (9):e319.

216. Dasgupta K: Sex differences in the development of higher systolic blood pressure during adolescence. Cardiology Review 2008, 25(5):54-7.

217. Lazarou C, Panagiotakos DB, Matalas AL, Lazarou C, Panagiotakos DB, Matalas AL: Lifestyle factors are determinants of children's blood pressure levels: the CYKIDS study. J Hum Hypertens 2009, 23(7):456-63.

218. Aman J, Skinner TC, de Beaufort CE, Swift PG, Aanstoot HJ, Cameron F, Hvidoere Study Group on Childhood Diabetes: Associations between physical activity, sedentary behavior, and glycemic control in a large cohort of adolescents with type 1 diabetes: the Hvidoere Study Group on Childhood Diabetes. Pediatr Diabetes 2009, 10(4):234-9.

219. Overby NC, Margeirsdottir HD, Brunborg C, Anderssen SA, Andersen LF, hlJorgensen K, Norwegian Study Group for Childhood Diabetes: Physical activity and overweight in children and adolescents using intensified insulin treatment. Pediatr Diabetes 2009, 10(2):135-41.

220. Mark $A E$, Janssen I: Relationship between screen time and metabolic syndrome in adolescents. J Public Health (Oxf) 2008, 30(2):153-60.

221. Robinson TN, Killen JD, Kraemer HC, Wilson DM, Matheson DM, Haskell WL, Pruitt LA, Powell TM, Owens AS, Thompson NS, Flint-Moore NM, Davis GJ, Emig KA, Brown RT, Rochon J, Green S, Varady A: Dance and reducing television viewing to prevent weight gain in African-American girls: the Stanford GEMS pilot study. Ethn Dis 2003, 13(Suppl 1):S65-77.

222. Holder MD, Coleman B, Sehn ZL, Holder MD, Coleman B, Sehn ZL: The contribution of active and passive leisure to children's well-being. Health Psychol 2009, 14(3):378-86.

223. Jackson LA, Zhao Y, Witt EA, Fitzgerald HE, Von EA, Harold R: Self-concept, self-esteem, gender, race, and information technology use. Cyberpsychol Behav 2009, 12(4):437-40.

224. Neumark-Sztainer D, Goeden C, Story M, Wall M: Associations between body satisfaction and physical activity in adolescents: Implications for programs aimed at preventing a broad spectrum of weight-related disorders. Eating Disorders 2004, 12(2):125-37.

225. Dominick JR: Videogames, television violence, and aggression in teenagers. J Commun 1984, 34(2):136-47.

226. Ohannessian CM: Media use and adolescent psychological adjustment: An examination of gender differences. J Child Fam Stud 2009, 18(5):583-93.

227. Wiggins JD: Self-esteem, earned grades, and television viewing habits of students. School Counselor 1987, 35(2):128-133.

228. Gortmaker SL, Salter CA, Walker DK, Dietz WH: The impact of television viewing on mental aptitude and achievement: A longitudinal study. Public Opinion Quarterly 1990, 54(4):Win-604

229. Mistry KB, Minkovitz CS, Strobino DM, Borzekowski DL: Children's television exposure and behavioral and social outcomes at 5.5 years: does timing of exposure matter? Pediatrics 2007, 120(4):762-9.

230. Kuntsche E, Pickett W, Overpeck M, Craig W, Boyce W, de Matos MG: Television viewing and forms of bullying among adolescents from eight countries. J Adolesc Health 2006, 39(6):908-15. 
231. Ussher MH, Owen CG, Cook DG, Whincup PH: The relationship between physical activity, sedentary behaviour and psychological wellbeing among adolescents. Soc Psychiatry Psychiatr Epidemiol 2007, 42(10):851-6.

232. Chowhan J, Stewart JM: Television and the behaviour of adolescents: does socio-economic status moderate the link? Soc Sci Med 2007, 65(7):1324-36.

233. Nelson MC, Gordon-Larsen P: Physical activity and sedentary behavior patterns are associated with selected adolescent health risk behaviors. Pediatrics 2006, 117(4):1281-90

234. Page RM, Zarco EP: Relationship between television viewing frequency and scores on shyness among Philippine high school students. Psychol Rep 2001, 89(2):366-8.

235. Brodersen NH, Steptoe A, Williamson S, Wardle J: Sociodemographic, developmental, environmental, and psychological correlates of physical activity and sedentary behavior at age 11 to 12 . Ann Behav Med 2005, 29(1):2-11.

236. Sakamoto A: Video game use and the development of sociocognitive abilities in children: Three surveys of elementary school students. J Appl Soc Psychol 1994, 24(1):21-42.

237. Ennemoser M, Schneider W: Relations of television viewing and reading: Findings from a 4-year longitudinal study. J Educ Psychol 2007, 99(2):349-68.

238. Lonner WJ, Thorndike RM, Forbes NE, Ashworth C: The influence of television on measured cognitive abilities: A study with Native Alaskan children. J Cross Cult Psychol 1985, 16(3):355-80.

239. Shin N: Exploring pathways from television viewing to academic achievement in school age children. J Genet Psychol 2004, 165(4):367-81.

240. nastassea-Vlachou K, Fryssira-Kanioura H, Papathanasiou-Klontza D, XipolitaZachariadi A, Matsaniotis N: The effects of television viewing in Greece, and the role of the paediatrician: a familiar triangle revisited. Eur J Pediatr 1996, 155(12):1057-60.

241. Jaruratanasirikul S, Wongwaitaweewong K, Sangsupawanich P: Electronic game play and school performance of adolescents in southern Thailand. Cyberpsychol Behav 2009, 12(5):509-12.

242. Kovacs FM, Del Real MTG, Gestoso M, Lopez J, Mufraggi N, Palou P: Relació entre hàbits de vida i qualificacions escolars en adolescents. Apunts Medicina de l'Esport 2008, 43(160):181-8, Spanish.

243. Kristjansson AL, Sigfusdottir ID, Allegrante JP, Helgason AR: Adolescent health behavior, contentment in school, and academic achievement. Am J Health Behav 2009, 33(1):69-79.

244. Sharif I, Sargent JD: Association between television, movie, and video game exposure and school performance. Pediatrics 2006, 118(4):1061-70

245. Caldas SJ, Bankston Cl: Black and white TV: Race, television viewing and academic achievement. Sociological Spectrum 1999, 19(1):39.

246. Shejwal B: Television viewing of higher secondary students: Does it affect their academic achievement and mathematical reasoning? Psychol Dev Soc J 2006, 18(2):201-13.

247. Cooper H, Valentine JC, Nye B, Lindsay JJ: Relationships between five after-school activities and academic achievement. J Educ Psychol 1999, 91(2):369-78.

248. Welch WW, Walberg HJ, Fraser BJ: Predicting elementary science learning using national assessment data. Journal of Research in Science Teaching 1986, 23(8):699-706.

249. Fetler M: Television viewing and school achievement. J Commun 1984, 34(2):104-18.

250. Roberts DF, Bachen CM, Hornby MC, Hernandez-Ramos P: Reading and television: Predictors of reading achievement at different age levels. Communication Research 1984, 11(1):9-49.

251. Walberg HJ, Tsai Sl: Reading achievement and diminishing returns to time. Journal of Educational Psychology 1984, 76(3):442-51.

252. Ridley-Johnson R, Cooper H, Chance J: The relation of children's television viewing to school achievement and I.Q. J Educ Res 1983, 76(5):294-7.

253. Walberg HJ, Weinstein $\mathrm{T}$ : The production of achievement and attitude in high school social studies. J Educ Res 1982, 75(5):285-93.

254. Scott LF: Relationships between elementary school children and television. J Educ Res 1958, 52:134-7.

255. Dumais SA: Cohort and gender differences in extracurricular participation: The relationship between activities, math achievement, and college expectations. Sociol Spectr 2009, 29(1):72.
256. Keith TZ, Reimers TM, Fehrmann PG, Pottebaum SM, Aubey LW: Parental involvement, homework, and TV time: Direct and indirect effects on high school achievement. J Edul Psychol 1986, 78(5):373-80.

257. Gaddy GD: Television's impact on high school achievement. Public Opin Q 1986, 50(3):340-59

258. Skoric MM, Teo LL, Neo RL: Children and video games: addiction, engagement, and scholastic achievement. Cyberpsychol Behav 2009, 12(5):567-72.

259. Jackson $L A$, Zhao Y, Kolenic A, Fitzgerald HE, Harold R, Von EA: Race, gender, and information technology use: the new digital divide. Cyberpsychol Behav 2008, 11(4):437-42.

260. Sharif I, Wills TA, Sargent JD: Effect of visual media use on school performance: A prospective study. J Adolesc Health 2010, 46(1):52-61

261. van Schie EG: Children and videogames: Leisure activities, aggression, social integration, and school performance. J Appl Soc Psychol 1997, 27(13):1175-94.

262. Hughes AR, Stewart L, Chapple J, McColl JH, Donaldson MD, Kelnar CJ, Zabihollah M, Ahmed F, Reilly JJ: Randomized, controlled trial of a bestpractice individualized behavioral program for treatment of childhood overweight: Scottish Childhood Overweight Treatment Trial (SCOTT). Pediatrics 2008, 121(3):e539-e546.

263. Shelton D, Le GK, Norton L, Stanton-Cook S, Morgan J, Masterman P: Randomized controlled trial: A parent-based group education program for overweight children. J Paediatr Child Health 2007, 43(12):799-805.

264. Goldfield GS, Mallory R, Parker T, Cunningham T, Legg C, Lumb A, Parker K, Prud'homme D, Adamo KB: Effects of open-loop feedback on physical activity and television viewing in overweight and obese children: a randomized, controlled trial. Pediatrics 2006, 118(1):e157-e166.

265. Epstein LH, Valoski AM, Vara LS, McCurley J, Wisniewski L, Kalarchian MA, Klein KR, Shrager LR: Effects of decreasing sedentary behavior and increasing activity on weight change in obese children. Health Psychol 1995, 14(2):109-15.

266. Tremblay MS, LeBlanc AG, Janssen I, Kho ME, Hicks A, Murumets K: Canadian sedentary behaviour guidelines for children and youth. Appl Physiol Nutr Metab 2011, 36(1):59-64.

267. Kho ME, Eva KW, Cook DJ, Brouwers MC: The Completeness of Reporting (CORE) index identifies important deficiencies in observational study conference abstracts. J Clin Epidemiol 2008, 61(12):1241-9.

268. Hopewell S, Clarke M, Askie L: Reporting of trials presented in conference abstracts needs to be improved. J Clin Epidemiol 2006, 59(7):681-4.

269. LeBlanc AG, Janssen I: Difference between self-reported and accelerometer measured moderate-to-vigorous physical activity in youth. Pediatr Exer Sci 2010, , 22: 223-34.

270. Adamo KB, Prince SA, Tricco AC, Connor-Gorber S, Tremblay MS: A comparison of indirect versus direct measures for assessing physical activity in the pediatric population: A systematic review. Int I Pediatr Obes 2009, 4(1):2-27.

271. Biddiss $\mathrm{E}$, Irwin J: Active video games to promote physical activity in children and youth. Arch Pediatr Adolesc Med 2010, 164(7):664-72.

doi:10.1186/1479-5868-8-98

Cite this article as: Tremblay et al.: Systematic review of sedentary behaviour and health indicators in school-aged children and youth. International Journal of Behavioral Nutrition and Physical Activity 2011 8:98.

\section{Submit your next manuscript to BioMed Central and take full advantage of:}

- Convenient online submission

- Thorough peer review

- No space constraints or color figure charges

- Immediate publication on acceptance

- Inclusion in PubMed, CAS, Scopus and Google Scholar

- Research which is freely available for redistribution

Submit your manuscript at www.biomedcentral.com/submit
C Biomed Central 